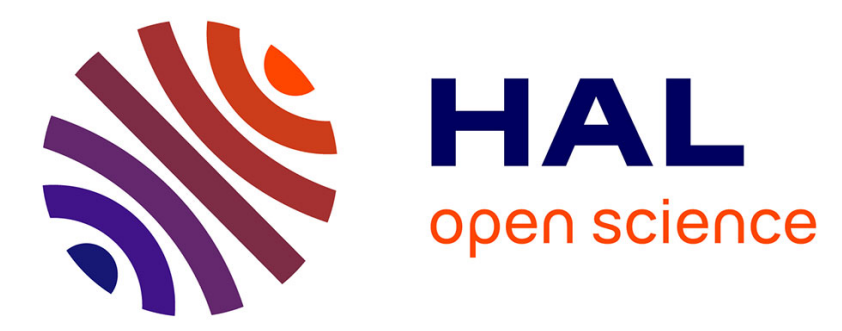

\title{
Computational micro to macro transitions for shape memory alloy composites using periodic homogenization
}

Georges Chatzigeorgiou, Yves Chemisky, Fodil Meraghni

\section{To cite this version:}

Georges Chatzigeorgiou, Yves Chemisky, Fodil Meraghni. Computational micro to macro transitions for shape memory alloy composites using periodic homogenization. Smart Materials and Structures, 2015, 24 (3), pp.1-17. 10.1088/0964-1726/24/3/035009 . hal-01196237

\section{HAL Id: hal-01196237 \\ https://hal.science/hal-01196237}

Submitted on 9 Sep 2015

HAL is a multi-disciplinary open access archive for the deposit and dissemination of scientific research documents, whether they are published or not. The documents may come from teaching and research institutions in France or abroad, or from public or private research centers.
L'archive ouverte pluridisciplinaire HAL, est destinée au dépôt et à la diffusion de documents scientifiques de niveau recherche, publiés ou non, émanant des établissements d'enseignement et de recherche français ou étrangers, des laboratoires publics ou privés. 


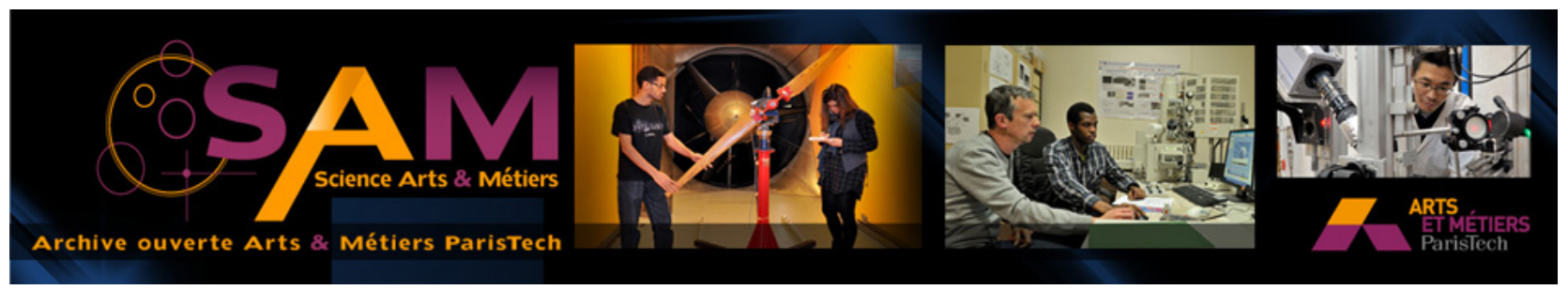

Science Arts \& Métiers (SAM)

is an open access repository that collects the work of Arts et Métiers ParisTech researchers and makes it freely available over the web where possible.

This is an author-deposited version published in: http://sam.ensam.eu

Handle ID: .http://hdl.handle.net/10985/9968

\section{To cite this version :}

George CHATZIGEORGIOU, Yves CHEMISKY, Fodil MERAGHNI - Computational micro to macro transitions for shape memory alloy composites using periodic homogenization - Smart Materials and Structures - Vol. 24, n³, p.1-17- 2015 


\title{
Computational micro to macro transitions for shape memory alloy composites using periodic homogenization
}

\author{
George Chatzigeorgiou ${ }^{1}$, Yves Chemisky and Fodil Meraghni \\ LEM3-UMR 7239 CNRS, Arts et Métiers ParisTech Metz-Lorraine, 4 Rue Augustin Fresnel 57078 Metz,
} France

\begin{abstract}
In the current manuscript, a homogenization framework is proposed for periodic composites with shape memory alloy (SMA) constituents under quasi-static thermomechanical conditions. The methodology is based on the step-by-step periodic homogenization, in which the macroscopic and the microscopic problems of the composite are solved simultaneously. The implementation of the framework is examined with numerical examples on SMA composite laminates.

Complexity of the composite nonlinear response and non-proportional stress state in the SMA appears, even in the case of uniaxial macroscopic boundary conditions. Moreover, under certain conditions, the composite laminate can exhibit a non-convex transformation surface.

Additionally, the transformation temperatures at various stress levels under isobaric thermal cycling can be quite different between the composite and the pure SMA.
\end{abstract}

\section{Introduction}

Shape memory aloys (SMAs) have been investigated for engineering applications for more than 40 years due to their ability to recover substantial deformation. The phenomenological modeling of the reversible martensitic transformation in SMAs has been well studied in the literature and several constitutive models have been proposed (Tanaka et al 1986, Liang and Rogers 1990, Brinson 1993, Boyd and Lagoudas 1996, Patoor et al 1996, Leclercq and Lexcellent 1996, Auricchio et al 1997, Anand and Gurtin 2003, Paiva et al 2005, Sadjadpour and Bhattacharya 2007, Hartl et al 2010, Chemisky et al 2011, Lagoudas et al 2012). Thorough reviews of the most common models and comparisons between them can be found in the works of Birman (1997), Patoor et al (2006), Lagoudas et al (2006), Paiva and Savi (2006). While these materials are considered good candidates for actuators, their relatively high cost restrains their usage in many industrial applications. Thus, alternative, less expensive solutions are investigated, including composites with SMA components.

The homogenization theory of media with periodic structure is a well-established theory (Bensoussan et al 1978, Sanchez-Palencia 1978, Tartar 1978, Allaire 1992, Murat and Tartar 1997). Modeling the mechanical behavior of elastoplastic, viscoplastic, or damaged composite materials requires advanced micromechanics and homogenization techniques from both mathematical and computational points of view (Suquet 1987, Ponte-Castañeda and Suquet 1997, Terada and Kikuchi 2001, Desrumaux et al 2001, Meraghni et al 2002, Jendli et al 2009, Mercier and Molinari 2009, Khatam and Pindera 2009, Tekoğlu and Pardoen 2010, Cavalcante et al 2011, Kruch and Chaboche 2011, Lahellec and Suquet 2013, Tsalis et al 2013). Reviews of different multiscale approaches for linear and nonlinear composites are 
available in the literature (Kanouté et al 2009, Pindera et al 2009, Charalambakis 2010, Geers et al 2010).

Recently, much research effort has focused on modeling the behavior of SMA composites. Porous SMAs have been extensively studied during the last two decades (Qidwai et al 2001, Entchev and Lagoudas 2002, 2004, Nemat-Nasser et al 2005, Panico and Brinson 2008). Moreover, in the literature, certain studies on composite beams with SMA reinforcement (Baz and Ro 1992), composite plates with SMA fibers (Rogers et al 1991, Ro and Baz 1995), and strips (Lagoudas et al 1997) have been reported. Micromechanical analyses on composites with SMA reinforcement have been performed by Boyd and Lagoudas (1994), Lagoudas et al (1994), Cherkaoui et al (2000), Wang and Shen (2000), Song and Sun (2000), Marfia (2005), Collard et al (2008), Lester et al (2011) using the self-consistent and/or Mori-Tanaka methods and by Kawai et al (1999) using the method of cells. The Mori-Tanaka method has also been utilized to study SMAs with precipitates (Piotrowski et al 2012). While these techniques allow us to obtain analytical or semi-analytical solutions, they can provide quite accurate results only under certain conditions: a) geometrical limitations, dealing only with matrix/ellipsoidal inclusion material systems and b) the Eshelby solution is no longer valid when the SMA is considered to be the matrix of the composite. The use of periodic homogenization in SMA composites has been reported in Herzog and Jacquet (2007), but in this methodology the elastic properties of the SMA were assumed to be constant, independent of the martensite volume fraction. Indeed, the approach proposed by the authors cannot be applied when the elastic properties vary with the martensite volume fraction. In the present work, such limitation is addressed, by following a different approach based on the tangent moduli of the material constituents.

The current work introduces a computational approach for composites with SMA components, based on the periodic homogenization theory. The advantage of this approach is that it can be applied to periodic media with SMAs independent of the material constituents geometry and the choice of the SMA constitutive law. The incremental step-by-step homogenization methodology solves the problem in both the micro- and macroscale simultaneously, which is very important, especially in the cases of nonlinear materials. The computational approach is utilized in the case of composite laminates consisting of SMAs undergoing martensite-austenite transformation and elastic or elastoplastic materials. The obtained results show some interesting characteristics of the overall composite behavior, like non-convex transformation surfaces under certain conditions.

The organization of the paper is as follows: sections 2 and 3 present the main concepts of homogenization for nonlinear periodic media and the computational scheme based on the return mapping algorithm technique. In section 4 the Lagoudas et al (2012) model, used in the numerical examples, is briefly described. The numerical studies of SMA laminate (multilayered) composites in sections 5 and 6 demonstrate the capabilities and highlights of the developed approach. Conclusions are presented in section 7 . For the completeness of

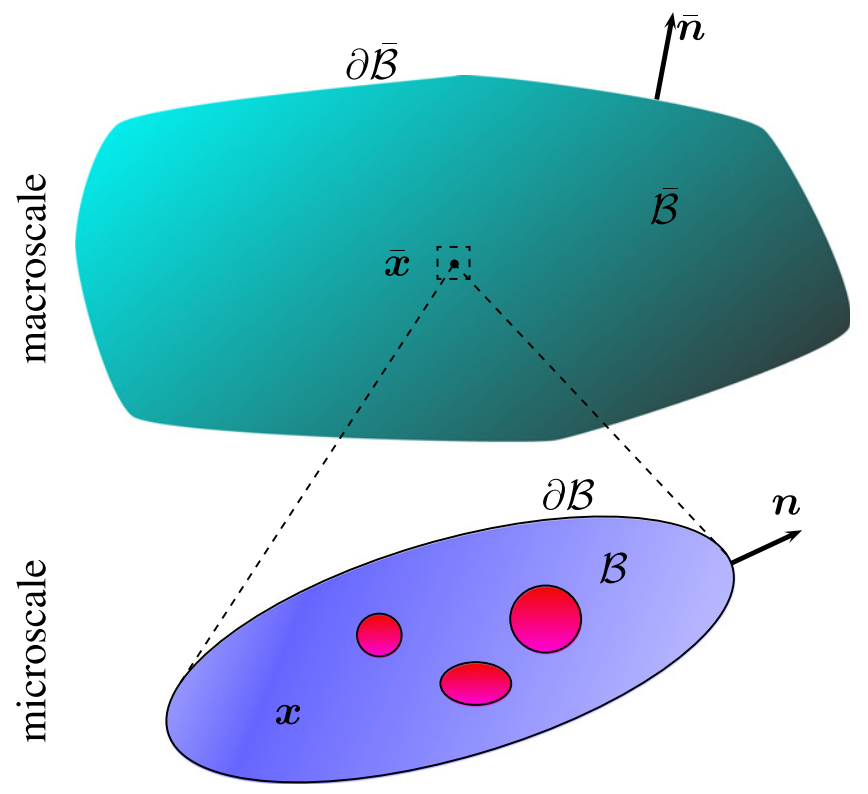

Figure 1. Schematic presentation of composite: macro- and microscale.

the paper, two appendices are included, which present the algorithms of the computational scheme and the semi-analytical solution details for the case of composite laminates.

\section{Theoretical framework of homogenization}

The principles of periodic homogenization have already introduced in the pioneering works of Bensoussan et al (1978), Sanchez-Palencia (1978), and for elastoplastic composites in Suquet (1987). Here the main points of the theory are briefly summarized.

In periodic homogenization a composite is described through the introduction of two suitable scales. The first scale, the microscopic, represents the microstructure considering different material constituents and their geometry. The second scale, the macroscopic, considers the overall body as an imaginary homogeneous medium (figure 1). The properties of this hypothetical homogeneous medium are identified through the solution of the microscale problem.

At the macroscale, the continuum body occupies the space $\overline{\mathcal{B}}$ with volume $\bar{V}$ and bounded by a surface $\partial \overline{\mathcal{B}}$ with normal unit vector $\overline{\boldsymbol{n}}$. Each macroscopic point is assigned with a position vector $\overline{\boldsymbol{x}}$ in $\overline{\mathcal{B}}$ (figure 1). On the other hand, at the microscale level, there is a representative volume element (RVE) that occupies the space $\mathcal{B}$ with volume $V$ and is bounded by a surface $\partial \mathcal{B}$ with normal unit vector $\boldsymbol{n}$. Each microscopic point is assigned with a position vector $\boldsymbol{x}$ in $\mathcal{B}$ (figure 1). In the sequel, a bar above a symbol $(\overline{(\bullet \bullet})$ denotes a macroscopic quantity or variable.

In the periodic homogenization framework, the two scales $\overline{\boldsymbol{x}}$ (overall scale) and $\boldsymbol{x}$ (microscale) are connected by a characteristic length $\varepsilon$ through the relation $x=\frac{\bar{x}}{\varepsilon}$. This characteristic length needs to be very small, tending to zero (i.e., the microstructure should be extremely small compared 
to the size of the composite), for the homogenization method to be accurate. Some typical examples of materials with periodic microstructure that meet these requirements are particulate-reinforced, aligned fiber, multilayered, and woven fabric composite.

In the following subsections the main principles that govern the two scales and the correlation between them are described. It is worth mentioning that the current work deals with quasi-static processes and considers the influence of the temperature in the composite's mechanical response. The spatial distribution of the temperature inside the material would require a fully coupled thermomechanical analysis, accounting for latent heat and thermal conduction effects, which exceeds the scope of this contribution. Thus, in all the studied numerical examples the temperature profile is considered known (i.e., spatially uniform temperature conditions).

\subsection{Microscale}

Since each RVE represents a single point in the macroscopic body, every microscopic variable is a function of both the macroscopic position of the specific RVE, $\bar{x}$, and the microscopic position, $\boldsymbol{x}$.

Upon mechanical loading, it is assumed that each microscopic point undergoes a displacement $\boldsymbol{u}$. In this paper small deformation processes are considered, thus the problem is formulated using small strain assumptions. For the kinematics on the RVE, the usual relation between the secondorder symmetric microscopic strain tensor $\boldsymbol{\epsilon}$ and the displacement vector $\boldsymbol{u}$ are considered,

$$
\begin{aligned}
\boldsymbol{\epsilon}(\overline{\boldsymbol{x}}, \boldsymbol{x})= & \frac{1}{2}[\operatorname{grad} \boldsymbol{u}(\overline{\boldsymbol{x}}, \boldsymbol{x}) \\
& \left.+[\operatorname{grad} \boldsymbol{u}(\overline{\boldsymbol{x}}, \boldsymbol{x})]^{\mathrm{t}}\right] \quad \text { in } \mathcal{B},
\end{aligned}
$$

where the superscript $t$ denotes the transpose of a matrix and $\operatorname{grad}\{\bullet\}=\frac{\partial\{\bullet\}}{\partial x}$. Ignoring micro-body forces and inertia effects, the micro-equilibrium is written

$$
\operatorname{div} \boldsymbol{\sigma}(\overline{\boldsymbol{x}}, \boldsymbol{x})=\mathbf{0} \quad \text { in } \mathcal{B},
$$

where $\boldsymbol{\sigma}$ is the second-order symmetric microscopic stress tensor, $\operatorname{div}\{\bullet\}=\operatorname{grad}\{\bullet\}: \boldsymbol{i}$, and $\boldsymbol{i}$ is the second-order identity tensor. For materials with thermomechanical response, such as SMAs, the microscale constitutive relation can be written in incremental form as (Lagoudas 2008)

$$
\begin{aligned}
\delta \boldsymbol{\sigma}(\overline{\boldsymbol{x}}, \boldsymbol{x})= & \boldsymbol{D}^{\epsilon}(\overline{\boldsymbol{x}}, \boldsymbol{x}): \delta \boldsymbol{\epsilon}(\overline{\boldsymbol{x}}, \boldsymbol{x}) \\
& +\boldsymbol{D}^{\theta}(\overline{\boldsymbol{x}}, \boldsymbol{x}) \delta \bar{\theta}(\overline{\boldsymbol{x}})
\end{aligned}
$$

where $\boldsymbol{D}^{\epsilon}$ is the symmetric fourth-order micro-tangent stiffness tensor, $\boldsymbol{D}^{\theta}$ is the symmetric second-order micro-tangent thermal modulus tensor and $\bar{\theta}$ is the macroscopic temperature. Such a relation implies that the temperature inside the RVE is assumed to be constant when considering the microscale stress-strain constitutive relations (see Ene 1983, Maghous and Creus 2003).
Finally, the increment of the mechanical micro-energy density function $W$ is written as

$$
\delta W(\overline{\boldsymbol{x}}, \boldsymbol{x})=\boldsymbol{\sigma}(\overline{\boldsymbol{x}}, \boldsymbol{x}): \delta \boldsymbol{\epsilon}(\overline{\boldsymbol{x}}, \boldsymbol{x}) .
$$

\subsection{Macroscale}

When the overall body is studied, every macroscopic variable is considered as a function only of the macroscopic position $\overline{\boldsymbol{x}}$.

A main assumption in the homogenization theory is that the macroscopic body is represented by a similar system of equations with the RVE. Thus, each macroscopic point upon mechanical loading undergoes a displacement $\overline{\boldsymbol{u}}$ and the deformation is characterized by the second-order symmetric macroscopic strain $\overline{\boldsymbol{\epsilon}}$, which is connected with the macrodisplacement $\overline{\boldsymbol{u}}$ through a relation of the form

$$
\overline{\boldsymbol{\epsilon}}(\overline{\boldsymbol{x}}):=\frac{1}{2}\left[\overline{\operatorname{grad}} \overline{\boldsymbol{u}}(\overline{\boldsymbol{x}})+[\overline{\operatorname{grad}} \overline{\boldsymbol{u}}(\overline{\boldsymbol{x}})]^{\mathrm{t}}\right] \text { in } \overline{\mathcal{B}},
$$

where $\overline{\operatorname{grad}}\{\bullet\}=\frac{\partial\{\bullet\}}{\partial \bar{x}}$. Under quasi-static loadings and ignoring macro-body forces the macro-equilibrium is expressed as

$$
\overline{\operatorname{div}} \overline{\boldsymbol{\sigma}}(\overline{\boldsymbol{x}})=\mathbf{0} \quad \text { in } \overline{\mathcal{B}},
$$

where $\bar{\sigma}$ is the second-order symmetric macroscopic stress tensor and $\overline{\operatorname{div}}\{\bullet\}=\overline{\operatorname{grad}}\{\bullet\}: \boldsymbol{i}$. Moreover, the constitutive relation that connects the macro-stresses with the macrostrains and macro-temperature is expressed in incremental form as

$$
\delta \overline{\boldsymbol{\sigma}}=\overline{\boldsymbol{D}}^{\epsilon}(\overline{\boldsymbol{x}}): \delta \overline{\boldsymbol{\epsilon}}(\overline{\boldsymbol{x}})+\overline{\boldsymbol{D}}^{\theta}(\overline{\boldsymbol{x}}) \delta \bar{\theta}(\overline{\boldsymbol{x}}),
$$

where $\overline{\boldsymbol{D}}^{\epsilon}$ and $\overline{\boldsymbol{D}}^{\theta}$ are the symmetric fourth-order macrotangent stiffness tensor and the symmetric second-order macro-tangent thermal modulus tensor respectively.

Finally, the increment of the macro-energy density function $\bar{W}$ is written as

$$
\delta \bar{W}(\overline{\boldsymbol{x}})=\overline{\boldsymbol{\sigma}}(\overline{\boldsymbol{x}}): \delta \overline{\boldsymbol{\epsilon}}(\overline{\boldsymbol{x}}) .
$$

\subsection{Connection between scales in periodic media}

The standard methodology in homogenization theories is to connect macroscopic quantities through volume averaging of their microscopic counterparts over the RVE (Hill 1967). Thus the relation between the macroscopic and microscopic strains is given by (Nemat-Nasser and Hori 1999)

$$
\begin{aligned}
\overline{\boldsymbol{\epsilon}}(\overline{\boldsymbol{x}})= & \frac{1}{V} \int_{\mathcal{B}} \boldsymbol{\epsilon}(\overline{\boldsymbol{x}}, \boldsymbol{x}) \mathrm{d} V \\
= & \frac{1}{2 V} \int_{\partial \mathcal{B}}[\boldsymbol{u}(\overline{\boldsymbol{x}}, \boldsymbol{x}) \otimes \boldsymbol{n}(\boldsymbol{x}) \\
& +\boldsymbol{n}(\boldsymbol{x}) \otimes \boldsymbol{u}(\overline{\boldsymbol{x}}, \boldsymbol{x})] \mathrm{d} S,
\end{aligned}
$$

and the relation between the macroscopic and microscopic 
stresses is expressed as (Qu and Cherkaoui 2006)

$$
\begin{aligned}
\overline{\boldsymbol{\sigma}}(\overline{\boldsymbol{x}}) & =\frac{1}{V} \int_{\mathcal{B}} \boldsymbol{\sigma}(\overline{\boldsymbol{x}}, \boldsymbol{x}) \mathrm{d} V \\
& =\frac{1}{V} \int_{\partial B} \boldsymbol{t}(\overline{\boldsymbol{x}}, \boldsymbol{x}) \otimes \boldsymbol{x} \mathrm{d} S,
\end{aligned}
$$

where $\boldsymbol{t}=\boldsymbol{\sigma} \cdot \boldsymbol{n}$ is the micro-traction vector. The second expressions of equations (9) and (10) are obtained with the help of the divergence theorem and the relations (1) and (2).

The periodic nature of the RVE is taken into account by considering that the micro-displacements $\boldsymbol{u}$ are expressed as the sum of a macroscopic (constant in the RVE) term, a linear term dependent on the macro-strain, and a periodic term (Suquet 1987)

$$
\begin{aligned}
\boldsymbol{u}(\overline{\boldsymbol{x}}, \boldsymbol{x})= & \boldsymbol{u}^{0}(\overline{\boldsymbol{x}})+\overline{\boldsymbol{\epsilon}}(\overline{\boldsymbol{x}}) \cdot \boldsymbol{x}+\boldsymbol{z}(\overline{\boldsymbol{x}}, \boldsymbol{x}), \\
& \boldsymbol{z}(\overline{\boldsymbol{x}}, \boldsymbol{x}) \text { periodic function with regard to } \boldsymbol{x} .
\end{aligned}
$$

In addition antiperiodicity conditions are considered for the micro-tractions, i.e.,

$$
\boldsymbol{t}(\overline{\boldsymbol{x}}, \boldsymbol{x}) \text { antiperiodic function with regard to } \boldsymbol{x} .
$$

Equations (1) and (11) lead to

$$
\begin{aligned}
\boldsymbol{\epsilon}(\overline{\boldsymbol{x}}, \boldsymbol{x})= & \overline{\boldsymbol{\epsilon}}(\overline{\boldsymbol{x}})+\frac{1}{2}[\operatorname{grad} z(\overline{\boldsymbol{x}}, \boldsymbol{x}) \\
& \left.+[\operatorname{grad} z(\overline{\boldsymbol{x}}, \boldsymbol{x})]^{\mathrm{t}}\right] .
\end{aligned}
$$

Following the classical procedure (see Suquet 1987), it can be proven that the usual Hill-Mandel condition holds, i.e.,

$$
\frac{1}{V} \int_{B} \delta W(\overline{\boldsymbol{x}}, \boldsymbol{x}) \mathrm{d} V=\delta \bar{W}(\overline{\boldsymbol{x}})
$$

\section{Numerical implementation of periodic homogenization}

Based on the principles of the periodic homogenization theory described in the previous section, a numerical procedure is required to obtain the response of both the microstructure and the overall body. The approach followed here has been proposed by Terada and Kikuchi (2001), Asada and Ohno (2007), Tsalis et al (2013) for the case of composites with elastoplastic constituents. A similar numerical scheme has also been implemented in the case of magnetomechanical composites under large deformations (Javili et al 2013). In this section, the essential points of the numerical procedure are presented.

A complete homogenization scheme requires the solution of the macroscale and the microscale simultaneously. In figure 2 an iterative scheme for homogenization is shown. From the macroscale analysis the macroscopic strains and temperatures are obtained, which are used in the RVE problem to compute the microscale variables and the correct macroscopic stresses. Moreover, the RVE problem provides information to compute the effective thermomechanical tangent moduli, which are used in the macroscale analysis. The proposed methodology is motivated from the return mapping

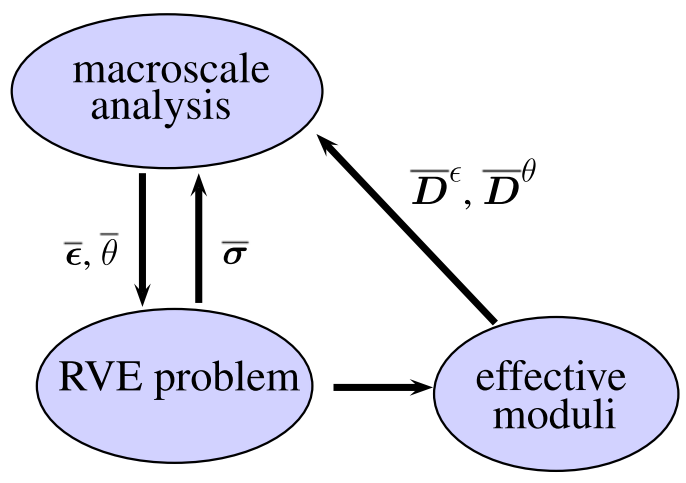

Figure 2. Computational homogenization scheme.

algorithm utilized to simulate the behavior of nonlinear materials (Simo and Hughes 1998).

In the sequel, the expressions are simplified by presenting the variables without mentioning their dependence on positions $\overline{\boldsymbol{x}}$ and/or $\boldsymbol{x}$.

\subsection{Macroscale problem}

At time step $n$ everything is considered to be known. Considering known temporal and spatial macroscopic temperature distribution throughout the composite, $\bar{\theta}$ at time step $n+1$ is also known. The task of the macroscale problem is to evaluate the macroscopic strains at time $n+1$.

By introducing the space of the macroscopic test functions

$$
\overline{\mathcal{V}}=\left\{\overline{\boldsymbol{\eta}}: \overline{\boldsymbol{\eta}}, \overline{\operatorname{grad}} \overline{\boldsymbol{\eta}} \in \mathcal{L}^{2}(\overline{\mathcal{B}}), \overline{\boldsymbol{\eta}}=\mathbf{0} \text { on } \partial \overline{\mathcal{B}}^{\mathrm{EB}}\right\}
$$

where $\partial \overline{\mathcal{B}}^{\mathrm{EB}} \subset \partial \overline{\mathcal{B}}$ denotes the part of the boundary surface where displacements are prescribed, the weak form of the macroscale problem is written as

$$
\begin{gathered}
\int_{\overline{\mathcal{B}}} \overline{\operatorname{grad}} \overline{\boldsymbol{\eta}}: \overline{\boldsymbol{\sigma}} \mathrm{d} V-\int_{\partial \overline{\mathcal{B}}^{\mathrm{NB}}} \overline{\boldsymbol{\eta}} \cdot \overline{\boldsymbol{t}} \mathrm{d} S=0, \\
\forall \overline{\boldsymbol{\eta}} \in \overline{\mathcal{V}} .
\end{gathered}
$$

In the last expression $\overline{\boldsymbol{t}}=\overline{\boldsymbol{\sigma}} \cdot \overline{\boldsymbol{n}}$ is the external traction, acting on the surface $\partial \overline{\mathcal{B}}^{\mathrm{NB}} \subset \partial \overline{\mathcal{B}}$, that the overall body is subjected to. Equation (16) generally represents a nonlinear system of equations. In such cases a Newton-Raphson scheme is utilized and the consistent linearization yields

$$
\begin{array}{r}
\int_{\overline{\mathcal{B}}} \overline{\operatorname{grad}} \overline{\boldsymbol{\eta}}:\left[\overline{\boldsymbol{\sigma}}^{(m)}+\overline{\boldsymbol{D}}^{\epsilon(m)}: \overline{\operatorname{grad}} \delta \overline{\boldsymbol{u}}^{(m)}\right] \mathrm{d} V \\
-\int_{\partial \overline{\mathcal{B}}^{\mathrm{NB}}} \overline{\boldsymbol{\eta}} \cdot \overline{\boldsymbol{t}} \mathrm{d} S=0, \quad \forall \overline{\boldsymbol{\eta}} \in \overline{\mathcal{V}},
\end{array}
$$

where $m$ denotes the iteration step. In the preceding expression the temperature does not appear, since it is known at time $n+1$ and thus it has no increment during the NewtonRaphson scheme. If the macroscopic stress $\overline{\boldsymbol{\sigma}}$ and the macrotangent modulus $\overline{\boldsymbol{D}}^{\epsilon}$ are known from the solution of the microscale problem, then equation (17) can be solved for $\delta \overline{\boldsymbol{u}}$ and thus the macro-strains $\overline{\boldsymbol{\epsilon}}$ at time step $n+1$ can be obtained. A detailed algorithmic scheme for the macroscale problem is provided in table A1 of appendix A. 


\subsection{Microscale problem}

Just as in the macroscale analysis, at time step $n$ everything is considered to be known. The task of the microscale problem is to evaluate the microscopic behavior at time $n+1$ and necessary quantities for the macroscale analysis. Specifically, the first objective is to obtain the microscopic strains $\boldsymbol{\epsilon}$ and the microscopic internal variables $\zeta$ (these can be, for example, the transformation strain tensor and the martensitic volume fraction in the case of the SMA material). The second objective is to obtain the macroscopic stresses and the macroscopic thermomechanical tangent moduli.

By introducing the space of the microscopic periodic test functions

$$
\begin{aligned}
\mathcal{V}= & \left\{\boldsymbol{\eta}: \boldsymbol{\eta}, \operatorname{grad} \boldsymbol{\eta} \in \mathcal{L}^{2}(\mathcal{B}),\right. \\
& \boldsymbol{\eta} \text { periodic on } \partial \mathcal{B}\}
\end{aligned}
$$

the weak form of the microscale problem is written as

$$
\int_{B} \operatorname{grad} \boldsymbol{\eta}: \boldsymbol{\sigma} \mathrm{d} V=0, \quad \forall \boldsymbol{\eta} \in \mathcal{V} .
$$

Equation (19) generally represents a nonlinear system of equations. In such cases a Newton-Raphson scheme is utilized and the consistent linearization yields

$$
\begin{aligned}
\int_{\mathcal{B}} \operatorname{grad} \boldsymbol{\eta}:\left[\boldsymbol{\sigma}^{\left(m^{*}\right)}+\boldsymbol{D}^{\epsilon\left(m^{*}\right)}: \operatorname{grad} \delta \boldsymbol{u}^{\left(m^{*}\right)}\right. \\
\left.+\boldsymbol{D}^{\theta\left(m^{*}\right)} \delta \overline{\boldsymbol{\theta}}^{\left(m^{*}\right)}\right] \mathrm{d} V=0, \quad \forall \boldsymbol{\eta} \in \mathcal{V},
\end{aligned}
$$

where $m^{*}$ denotes the iteration step. With the help of equation (11) the last relation is written

$$
\begin{aligned}
& \int_{\mathcal{B}} \operatorname{grad} \boldsymbol{\eta}:\left[\boldsymbol{\sigma}^{\left(m^{*}\right)}+\boldsymbol{D}^{\epsilon\left(m^{*}\right)}: \operatorname{grad} \delta \boldsymbol{z}^{\left(m^{*}\right)}\right. \\
& \left.+\boldsymbol{D}^{\epsilon\left(m^{*}\right)}: \delta \boldsymbol{\epsilon}^{\left(m^{*}\right)}+\boldsymbol{D}^{\theta\left(m^{*}\right)} \delta \bar{\theta}^{\left(m^{*}\right)}\right] \mathrm{d} V=0, \\
& \quad \forall \boldsymbol{\eta} \in \mathcal{V} .
\end{aligned}
$$

At this point a methodology similar to the return mapping algorithm is used to solve the problem.

1. As a first step the macro-strain and the macrotemperature are provided exclusively by the macroscale analysis, which means that the terms $\delta \overline{\boldsymbol{\epsilon}}$ and $\delta \bar{\theta}$ in equation (21) are zero. Thus

$$
\begin{gathered}
\int_{\mathcal{B}} \operatorname{grad} \boldsymbol{\eta}:\left[\boldsymbol{\sigma}^{\left(m^{*}\right)}+\boldsymbol{D}^{\epsilon\left(m^{*}\right)}: \operatorname{grad} \delta \boldsymbol{z}^{\left(m^{*}\right)}\right] \mathrm{d} V=0, \\
\forall \boldsymbol{\eta} \in \mathcal{V} .
\end{gathered}
$$

The material behavior of the constituents is described by appropriate constitutive law algorithms which, using the microscopic strain $\boldsymbol{\epsilon}$ and the microscopic internal variables $\zeta$ of the previous iteration step, can provide updated values of the internal variables, as well as the micro-stress $\boldsymbol{\sigma}$ and the micro-tangent moduli $\boldsymbol{D}^{\epsilon}, \boldsymbol{D}^{\theta}$ for the next iteration step (see Simo and Hughes 1998 for plastic and viscoplastic materials and Lagoudas 2008 for SMA materials). Thus, equation (22) constitutes a nonlinear system with regard to $\delta z$ that can be solved iteratively. Once the convergence is achieved (i.e., the residual term $\int_{\mathcal{B}} \operatorname{grad} \boldsymbol{\eta}: \boldsymbol{\sigma}^{\left(m^{*}\right)} \mathrm{d} V$ is close to zero), the macroscopic stress is obtained from equation (10). The complete algorithm of this first step (RVE problem) of the microscale analysis is given in table $\mathrm{A} 2$ of appendix A.

2. Once the first step is completed, the residual term is assumed exactly zero. The next step is to obtain the macroscopic tangent moduli that will allow us to proceed to an elastic prediction in the macroscale analysis. Thus, technically the terms $\delta \overline{\boldsymbol{\epsilon}}$ and $\delta \bar{\theta}$ are 'released' from being zero and equation (21) is written

$$
\begin{gathered}
\int_{\mathcal{B}} \operatorname{grad} \boldsymbol{\eta}:\left[\boldsymbol{D}^{\epsilon}: \operatorname{grad} \delta \boldsymbol{z}+\boldsymbol{D}^{\epsilon}: \delta \overline{\boldsymbol{\epsilon}}+\boldsymbol{D}^{\theta} \delta \bar{\theta}\right] \\
\mathrm{d} V=0, \forall \boldsymbol{\eta} \in \mathcal{V},
\end{gathered}
$$

where the micro-tangent moduli $\boldsymbol{D}^{\epsilon}, \boldsymbol{D}^{\theta}$ at each local micro-point are those obtained from the RVE problem after its convergence. Equation (23) has, up to a macroscopic constant, a solution of the form (Ene 1983)

$$
\delta z=\delta \bar{\epsilon}: \chi^{\epsilon}+\delta \bar{\theta} \chi^{\theta}
$$

In the preceding expression, $\chi^{\epsilon}$ is a third-order corrector tensor which is computed from the solution of the system

$$
\begin{gathered}
\int_{\mathcal{B}} \operatorname{grad} \boldsymbol{\eta}:\left[\boldsymbol{D}^{\epsilon}: \operatorname{grad} \chi^{\epsilon}+\boldsymbol{D}^{\epsilon}\right] \mathrm{d} V=0, \\
\forall \boldsymbol{\eta} \in \mathcal{V},
\end{gathered}
$$

where $\tilde{:}$ denotes the non-standard double contraction operation between two fourth-order tensors $\boldsymbol{L}$ and $\boldsymbol{M}$ with $[\boldsymbol{L}: \boldsymbol{M}]_{i j k l}=[\boldsymbol{L}]_{i j p q}[\boldsymbol{M}]_{k l p q}$. Additionally, $\chi^{\theta}$ is a vector corrector which is computed from the solution of the system

$$
\begin{gathered}
\int_{\mathcal{B}} \operatorname{grad} \boldsymbol{\eta}:\left[\boldsymbol{D}^{\epsilon}: \operatorname{grad} \chi^{\theta}+\boldsymbol{D}^{\theta}\right] \mathrm{d} V=0, \\
\forall \boldsymbol{\eta} \in \mathcal{V} .
\end{gathered}
$$

Using equations (3), and (10), (13), and (24) and considering the symmetries of the tensors $\boldsymbol{D}^{\epsilon}$ and $\boldsymbol{D}^{\theta}$, the increment of the macroscopic stress is expressed as

$$
\begin{aligned}
\delta \overline{\boldsymbol{\sigma}}= & \frac{1}{V} \int_{B} \delta \boldsymbol{\sigma} \mathrm{d} V=\frac{1}{V} \int_{B}\left[\boldsymbol{D}^{\epsilon}: \delta \boldsymbol{\epsilon}+\boldsymbol{D}^{\theta} \delta \bar{\theta}\right] \mathrm{d} V \\
= & \frac{1}{V} \int_{B}\left[\boldsymbol{D}^{\epsilon}: \operatorname{grad} \delta \boldsymbol{z}+\boldsymbol{D}^{\epsilon}: \delta \boldsymbol{\epsilon}+\boldsymbol{D}^{\theta} \delta \bar{\theta}\right] \mathrm{d} V \\
= & \frac{1}{V} \int_{B}\left[\boldsymbol{D}^{\epsilon}: \operatorname{grad} \chi^{\epsilon}+\boldsymbol{D}^{\epsilon}\right] \mathrm{d} V: \delta \overline{\boldsymbol{\epsilon}} \\
& +\frac{1}{V} \int_{B}\left[\boldsymbol{D}^{\epsilon}: \operatorname{grad} \chi^{\theta}+\boldsymbol{D}^{\theta}\right] \mathrm{d} V \delta \bar{\theta} .
\end{aligned}
$$

Comparing the last expression and equation (7) leads to

$$
\begin{aligned}
& \overline{\boldsymbol{D}}^{\epsilon}=\frac{1}{V} \int_{\mathcal{B}}\left[\boldsymbol{D}^{\epsilon}: \operatorname{grad} \chi^{\epsilon}+\boldsymbol{D}^{\epsilon}\right] \mathrm{d} V, \\
& \overline{\boldsymbol{D}}^{\theta}=\frac{1}{V} \int_{\mathcal{B}}\left[\boldsymbol{D}^{\epsilon}: \operatorname{grad} \chi^{\theta}+\boldsymbol{D}^{\theta}\right] \mathrm{d} V .
\end{aligned}
$$

The complete algorithm of this second step of the 
microscale analysis (effective tangent moduli problem) is given in table $\mathrm{A} 3$ of appendix A.

It is worth mentioning that, while the macroscopic thermal tangent modulus tensor can be calculated with the present approach, it does not affect the macroscopic solution of the problem, since it does not enter in the iterative NewtonRaphson scheme for the macro-displacement. This tensor is useful in fully coupled thermomecanical analyses.

\section{SMA constitutive law}

The homogenization framework presented in the previous sections is independent of the choice of the constitutive laws for the material constituents. To illustrate the methodology through numerical examples, a choice of a SMA constitutive model is required that accounts for the phase transformation between austenite and martensite. Here the recent and powerful phase transformation model by Lagoudas et al (2012) is chosen for two reasons: a) it is robust, widely utilized and thermodynamically consistent and b) it has the ability to account for three-dimensional loading cases.

According to Lagoudas et al (2012), the SMA Gibb's free energy functional depends on the stress $\boldsymbol{\sigma}$ and the temperature $T$, as well as on some internal variables. The chosen internal variables are the transformation strain $\boldsymbol{\epsilon}^{\text {tr }}$ and the martensitic volume fraction $\xi$. The total strain, $\boldsymbol{\epsilon}$, is decomposed in three parts, the elastic, $\boldsymbol{\epsilon}^{\mathrm{el}}$, the thermal $\boldsymbol{\epsilon}^{\text {th }}$, and the transformation strain, $\boldsymbol{\epsilon}^{\text {tr }}$,

$$
\boldsymbol{\epsilon}=\boldsymbol{\epsilon}^{\mathrm{el}}+\boldsymbol{\epsilon}^{\mathrm{th}}+\boldsymbol{\epsilon}^{\mathrm{tr}} .
$$

The elastic strain is connected with the stress though the linear relation

$$
\boldsymbol{\epsilon}^{\mathrm{el}}=S(\xi): \sigma .
$$

$\boldsymbol{S}(\xi)$ is the compliance tensor of the SMA, which depends on $\xi$ via a rule of mixtures relation

$$
S(\xi)=S^{A}+\xi \Delta S, \quad \Delta S=S^{M}-S^{A}
$$

where the superscripts $M$ and $A$ denote martensite and austenite respectively. For isotropic SMAs the compliance tensor is a function of the Young moduli $E^{A}, E^{M}$ and the Poisson ratios $\nu^{A}, \nu^{M}$. The thermal strain is given by

$$
\boldsymbol{\epsilon}^{\text {th }}=\alpha\left[\theta-\theta_{0}\right],
$$

where $\theta_{0}$ is a reference temperature and $\boldsymbol{\alpha}$ is the thermal expansion coefficient (same for austenite and martensite). With regard to the phase transformation, forward transformation is the process in which the austenite is transformed into martensite $(\dot{\xi}>0)$ and reverse transformation is the process in which the martensite is transformed into austenite $(\dot{\xi}<0)$. The transformation strain is provided through an evolution law of the form

$$
\dot{\boldsymbol{\epsilon}}^{\mathrm{tr}}=\Lambda \dot{\xi}, \quad \boldsymbol{\Lambda}= \begin{cases}\frac{3}{2} H^{\mathrm{cur}} \frac{\boldsymbol{\sigma}^{\prime}}{\sigma^{\mathrm{vM}}}, & \dot{\xi}>0, \\ \frac{\boldsymbol{\epsilon}^{t-r}}{\xi^{t-r}}, & \dot{\xi}<0,\end{cases}
$$

where $\boldsymbol{\sigma}^{\prime}=\boldsymbol{\sigma}-\frac{1}{3} \operatorname{trace}(\boldsymbol{\sigma}) \boldsymbol{i}$ is the deviatoric part of the stress tensor, $\sigma^{\mathrm{vM}}=\sqrt{\frac{3}{2} \sigma^{\prime}: \sigma^{\prime}}$ is the von Mises effective stress tensor, $\boldsymbol{\epsilon}^{t-r}$ is the transformation strain tensor at the end of the forward transformation, $\xi^{t-r}$ is the martensitic volume fraction at the end of the forward transformation (for complete transformation loops $\xi^{t-r}=1$ ), and $H^{\text {cur }}$ is a scalar material parameter associated with the maximum transformation strain and is connected with $\sigma^{\mathrm{vM}}$ through the relation

$$
\begin{aligned}
& H^{\text {cur }} \\
& =\left\{\begin{array}{l}
H^{\mathrm{min}}, \\
\sigma^{\mathrm{vM}} \leqslant \sigma^{\text {crit }}, \\
H^{\mathrm{min}}+\left[H^{\mathrm{sat}}-H^{\mathrm{min}}\right]\left[1-e^{-k\left[\sigma^{\mathrm{vM}}-\sigma^{\text {crit }}\right]}\right], \\
\sigma^{\mathrm{vM}}>\sigma^{\text {crit }} .
\end{array}\right.
\end{aligned}
$$

In the precedig expression, $H^{\mathrm{min}}, H^{\text {sat }}, \sigma^{\text {crit }}$, and $k$ are material parameters (for further details, see Lagoudas et al 2012).

Based on standard thermodynamics considerations, the model identifies the mechanical dissipation during forward and reverse transformation to be expressed as

$$
\pi \dot{\xi} \geqslant 0
$$

In the last expression $\pi$ is the thermodynamic force conjugate to $\xi$. Assuming the same thermal expansion coefficients and heat capacities for austenite and martensite, this force is written as

$$
\pi=\boldsymbol{\sigma}: \boldsymbol{\Lambda}+\frac{1}{2} \boldsymbol{\sigma}: \Delta \boldsymbol{S}: \boldsymbol{\sigma}+\rho \Delta s_{0}-\rho \Delta u_{0}-f,
$$

where $\rho$ is the density, $\Delta s_{0}$ is the change of the effective specific entropy between the austenite and martensite, $\Delta u_{0}$ is the change of the effective specific internal energy between the austenite and martensite, and $f$ is a smooth transformation hardening function,

$$
f= \begin{cases}\frac{1}{2} a_{1}\left[1+\xi^{n_{1}}-[1-\xi]^{n_{2}}\right]+a_{3}, & \dot{\xi}>0, \\ \frac{1}{2} a_{2}\left[1+\xi^{n_{3}}-[1-\xi]^{n_{4}}\right]-a_{3}, & \dot{\xi}<0 .\end{cases}
$$

In the preceding expression, $n_{1}-n_{4}$ are exponents related to the smoothness of the function and $a_{1}-a_{3}$ are material parameters connected to the two phase diagram slopes, $C^{A}$ and $C^{M}$, and the four transformation temperatures: martensitic start $M_{s}$, martensitic finish $M_{f}$, austenitic start $A_{s}$, and austenitic finish $A_{f}$ (for further details, see Lagoudas et al 2012). Both forward and reverse transformation processes are limited by the value of $\xi$, which should be between 0 and 1 . For the forward and reverse transformation processes appropriate plasticity-type functions $\Phi^{\text {forward }}$ and $\Phi^{\text {reverse }}$ are identified, accompanied by 
Kuhn-Tucker conditions,

$\dot{\xi} \geqslant 0, \quad \Phi^{\text {forward }}=\pi-Y \leqslant 0, \quad \dot{\xi} \Phi^{\text {forward }}=0$,

$\dot{\xi} \leqslant 0, \quad \Phi^{\text {reverse }}=-\pi-Y \leqslant 0, \quad \dot{\xi} \Phi^{\text {reverse }}=0$,

where $Y$ is a material parameter similar to the yield stress in plasticity.

Providing (a) the total strain $\boldsymbol{\epsilon}$ and temperature $\theta$ at time step $n+1$, (b) the transformation strain $\boldsymbol{\epsilon}^{\text {tr }}$ and (c) the martensitic volume fraction $\xi$ at time step $n$, the system of equations (29), (30), (33), and (38) can be solved iteratively through a numerical scheme (for instance the convex cutting plane return mapping algorithm) to identify the stress $\boldsymbol{\sigma}$, the transformation strain $\boldsymbol{\epsilon}^{\text {tr }}$ and the martensitic volume fraction $\xi$ at time step $n+1$. Once these quantities are known, the continuum tangent modulus $\boldsymbol{D}$ is computed and used for the next elastic prediction in the RVE problem. Additional details on the numerical implementation of the model can be found in Lagoudas (2008).

One disadvantage of the specific SMA model is that it cannot handle minor loops in the transformation regime. Thus, in all the proceeding analyses the loading and unloading conditions guarantee fully forward and fully reverse transformations, respectively.

\section{Composite laminate with SMA constituents: isothermal case}

This section studies the macroscopic behavior of a composite laminate, whose RVE includes an SMA material with volume fraction $c$ and another metallic material with volume fraction $1-c$ (figure 3(b)). In the following studied cases, the composite is subjected to a combination of a normal macroscopic stress $\bar{\sigma}_{11}$ in the $\bar{x}_{1}$ direction (normal to the layers) and a shear macroscopic stress $\bar{\sigma}_{12}$, as figure 3(a) depicts.

The RVE and the effective tangent modulus problems of a composite laminate are reduced to systems of one-dimensional differential equations and they have an analytical or semi-analytical solution (see for example Chatzigeorgiou et al 2014 in the case of composites with energetic interfaces). For manuscript completeness, appendix B presents the solutions of these problems for a laminate composite with $N$ layers exhibiting nonlinear behavior.

Due to the applied macroscopic stress boundary conditions, the complete homogenization problem needs to be solved iteratively and simultaneously in both micro- and macroscale levels, as the procedure described in section 3 indicates: The macroscopic boundary condition must be imposed incrementally. In the first step, the application of macroscopic stress leads to a triaxial normal and shear strain response. In the second step, this macroscopic strain tensor is implemented into the RVE problem to obtain the corrected macroscopic stress and the effective tangent modulus. The process continues iteratively until the corrected stress is approximately equal to the applied stress. Once this is achieved, the procedure continues with the next time increment.

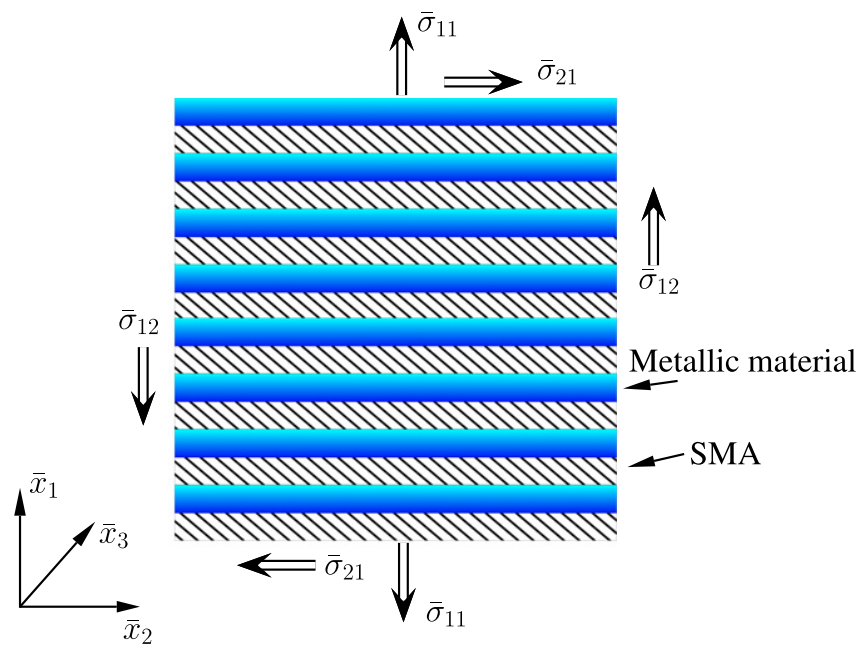

(a)

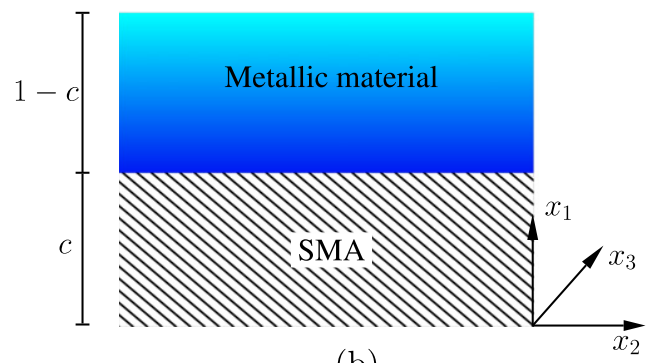

(b)

Figure 3. Schematic representation of (a) a multilayered material with SMA constituents under tension-shear loading and (b) the RVE of the composite.

Table 1. Material properties of SMA (Lagoudas 2008).

\begin{tabular}{lcc}
\hline Property & Austenite & Martensite \\
\hline Young modulus $(\mathrm{GPa})$ & 55 & 46 \\
Poisson ratio & 0.33 & 0.33 \\
$M_{s}(\mathrm{~K})$ & & 245 \\
$M_{f}(\mathrm{~K})$ & 270 & 230 \\
$A_{s}(\mathrm{~K})$ & 280 & \\
$A_{f}(\mathrm{~K})$ & \multicolumn{2}{c}{1} \\
$n_{1}, n_{2}$ & 1 & \\
$n_{3}, n_{4}$ & 7.4 & 7.4 \\
Phase diagram slope $(\mathrm{MPa} / \mathrm{K})$ & \multicolumn{2}{c}{0.056} \\
$H^{\text {min }}=H^{\text {sat }}$ & \multicolumn{2}{c}{} \\
\hline
\end{tabular}

In the numerical examples that follow, a SMA material is chosen, whose properties are summarized in table 1. For simplicity in obtaining the stress levels at the onset and finish of transformations, the smoothness of the hardening function has been removed by setting all the transformation exponents equal to 1 . Figure 4 shows the pure SMA pseudoelastic response at $300 \mathrm{~K}$ under uniaxial macroscopic loading and its phase diagram. All demonstrated results in the examples of this section are obtained for constant temperature, equal to $300 \mathrm{~K}$, and for zero thermal strains. The numerical examples have been performed using a developed c++ code, based on the theory and numerical scheme presented previously. 


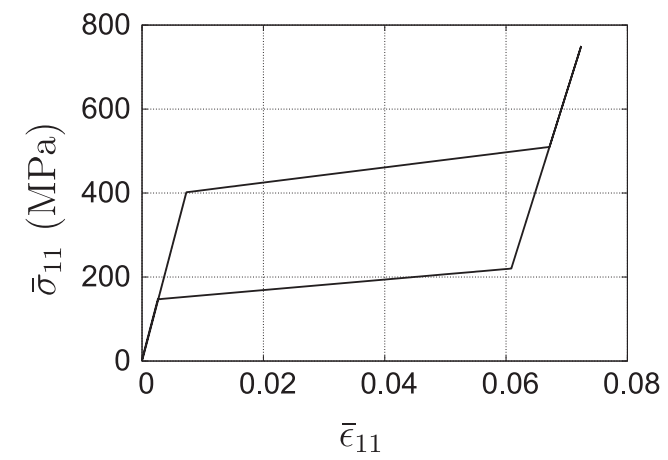

(a)

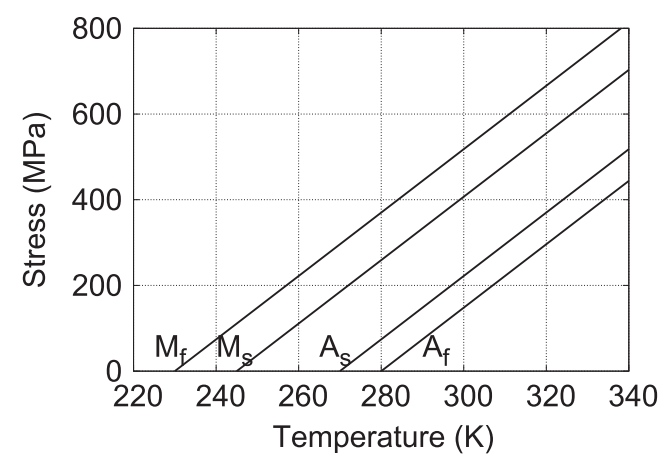

(b)

Figure 4. (a) SMA pseudoelastic response at $300 \mathrm{~K}$ under uniaxial tension loading-unloading conditions, (b) SMA phase diagram.

\subsection{Laminate with SMA and elastic material}

In the first series of examples the metallic material is assumed to be elastic with a Young modulus of $69 \mathrm{GPa}$ and a Poisson ratio of 0.3 . Figure 5 demonstrates the macroscopic stressstrain response of the multilayered composite when subjected to pure tension, for five different SMA volume fractions, 100 (pure SMA), 80, 60, 40 and 20\%. From these curves it is clearly observed that the decrease of the SMA volume fraction reduces the hysteresis of the composite response, and decreases the maximum transformation strain. Due to the elastic response of the metallic material, the stress levels required to complete the transformation of a composite with significant metallic material volume fraction are extremely high and thus other type of phenomena should also appear (development of plastic strains, damage, etc).

Another relevant observation is that even though the composite is under uniaxial stress state, the material constituents, at the RVE scale, are locally subjected to a nonproportional triaxial stress state (see table in figure 5, as well as figure 8). This could significantly affect the SMA response and would trigger other types of mechanisms, like martensitic variant reorientation, that the present SMA model cannot capture.

In figure 6 the macroscopic stress-strain response of the composite laminate when subjected to simple shear is presented. It is observed that the decrease of SMA content in the composite decreases the hysteretic response, but does not alter the stress levels that forward and reverse transformations start and finish.

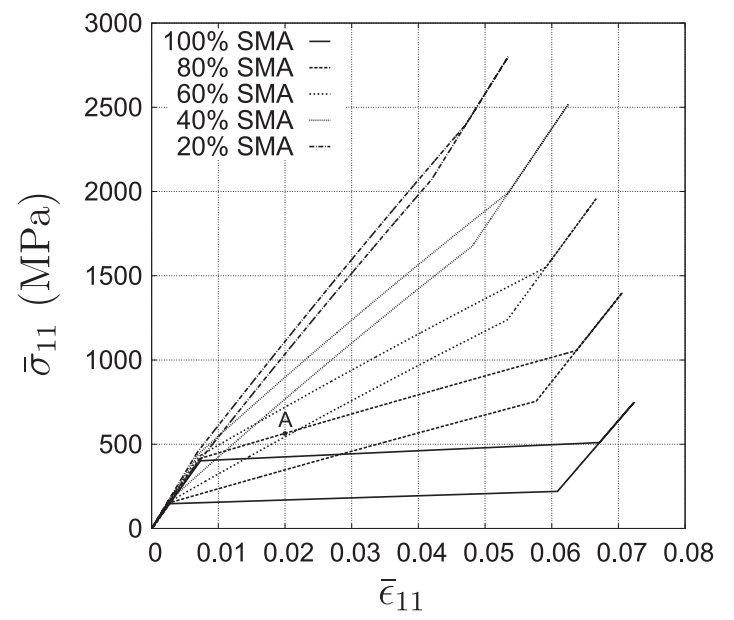

\begin{tabular}{|c|c|c|}
\hline \multicolumn{3}{|c|}{ Point A: composite with $80 \%$ SMA at 0.02} \\
macro-strain during forward transformation \\
\hline micro-stress $(\mathrm{MPa})$ & SMA & Metallic material \\
\hline$\sigma_{11}$ & 565 & 565 \\
\hline$\sigma_{22}$ & 140.31 & -561.22 \\
\hline$\sigma_{33}$ & 140.31 & -561.22 \\
\hline
\end{tabular}

Figure 5. Composite response under pure tension macro-loading at various SMA volume fractions and RVE response at specific macrostrain conditions.

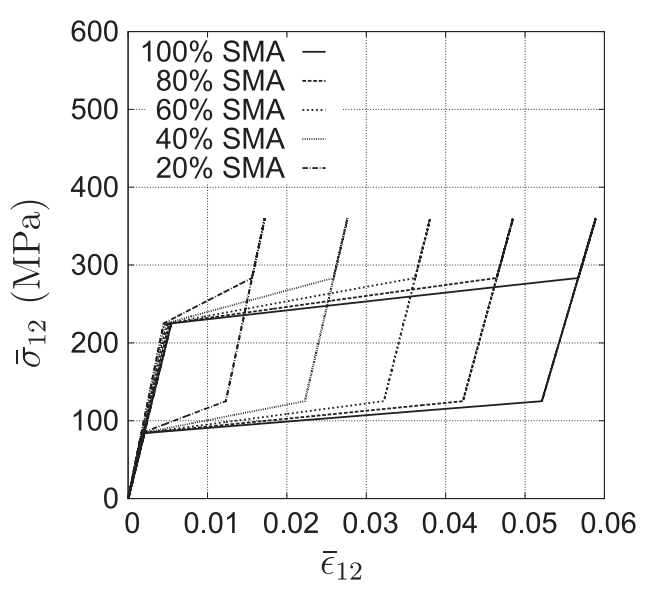

Figure 6. Composite response under simple shear macro-loading at various SMA volume fractions.

Figure 7 illustrates the transformation characteristics under combined shear-tension macro-loading conditions. Specifically, the decrease of SMA volume fraction in the composite does not seem to influence significantly the onset of forward transformation (figure 7(a)), but it causes drastic changes at the end of forward and the onset of reverse transformation (figures 7(b) and 7(c), respectively). Furthermore, it should be noticed that, for SMA volume fractions below $60 \%$, the end of reverse transformation presents a nonconvex response (figure $7(\mathrm{~d})$ ). This peculiar behavior arises as the result of the extreme non-proportionality in the stress 


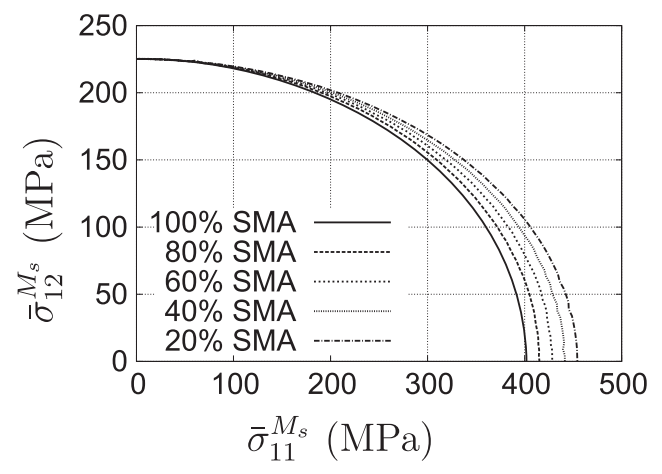

(a)

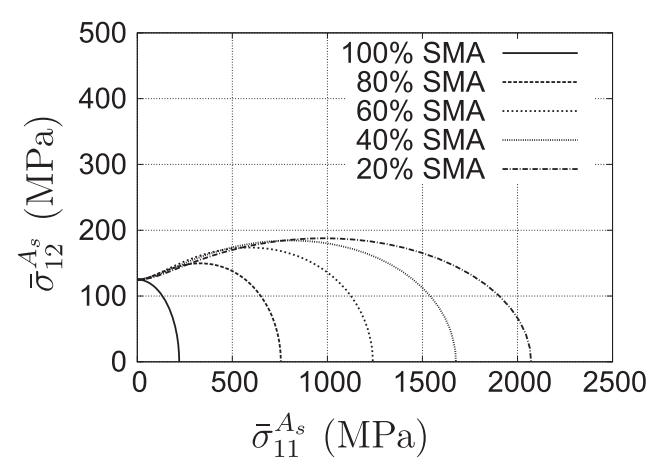

(c)

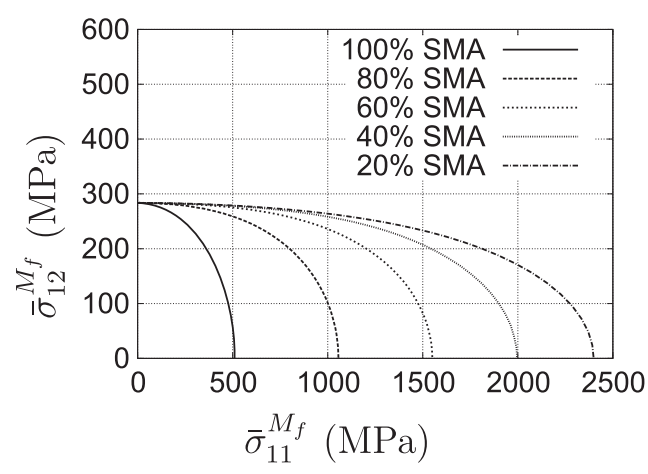

(b)

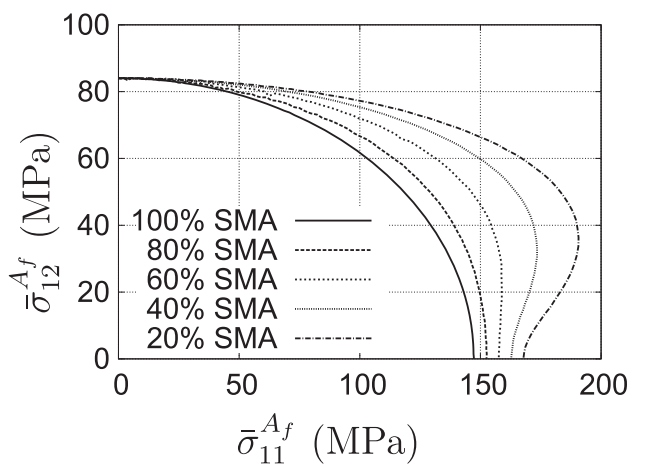

(d)

Figure 7. Shear versus normal macro-stress at (a) start of forward transformation, (a) finish of forward transformation, (c) start of reverse transformation, and (d) finish of reverse transformation.

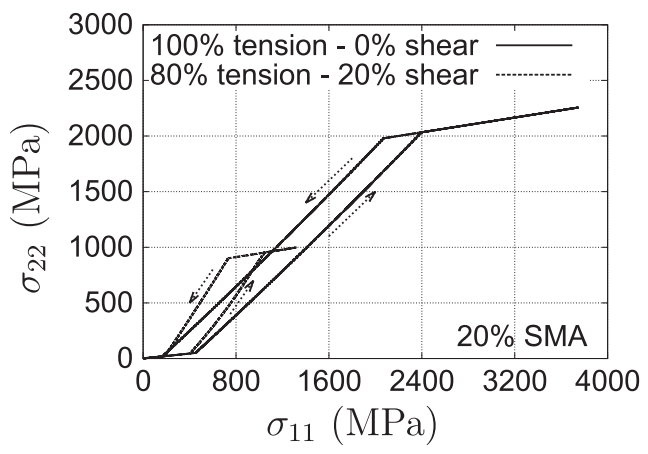

Figure 8. Multilayered composite with 20\% SMA volume fraction: normal micro-stresses inside the SMA for i) uniaxial macroscopic tension and ii) $80 \% \bar{\sigma}_{11}-20 \% \bar{\sigma}_{12}$.

response of the SMA. Figure 8 shows the micro-stresses in two directions (normal and parallel to the layers) inside the SMA constituent for a composite with $80 \%$ metallic material volume fraction. It is observed that, when the SMA is not transforming, the $\sigma_{11}$ is the dominant normal stress. During the transformation on the other hand, the incompressibility of the transformation strains and the loading requirement that the macroscopic stress $\bar{\sigma}_{22}$ must be zero cause a significant increase of the micro-stress $\sigma_{22}$ in the SMA leading to be almost equal (at the end of forward transformation), and even exceeding the normal stress $\sigma_{11}$ (at the beginning of reverse transformation). This alteration of the micro-stresses analogy causes a complicated loading path and, as figure 8 depicts, the finish of reverse transformation for two different macroscopic loading conditions (100\% tension and $80 \%$ tension-20\% shear) appears almost at the same stress level. So it becomes clear that even for simple macroscopic loading conditions, the microscopic response at each phase can be very complicated and strongly non-proportional. Thus, while the SMA constitutive law ensures a convex SMA response at all times (as figure 7 depicts for $0 \%$ metallic material), there is no guarantee that the average (overall) response of the composite will behave in the same way. The SMA constituent drives the start and finish of transformation of the composite, but the stress state of the two cannot be linked intuitively, and it is possible to observe a peculiar average response without violating the convexity of the materials in the microscale. In the occasion of low SMA content in the composite, the macroscopic response is driven mainly by the elastic material, while the transformation characteristics still depends on the SMA material, causing more disturbance in the overall transformation surfaces, compared to composites with high SMA content (figure 7).

Curves like the ones presented in figure 7 can be very useful for designing a phenomenological macroscopic model for the composite response. In such a model the material constants could be identified through an appropriate parameter identification method (Meraghni et al 2014). Of course, special attention needs to be taken when non-convex composite transformation surfaces appear. 


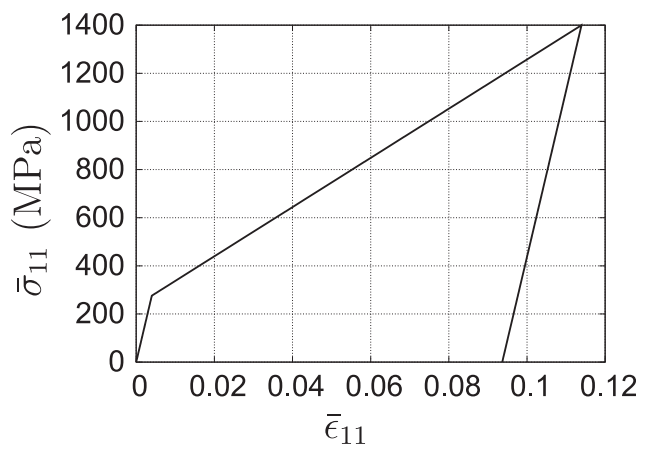

Figure 9. Metallic material elastoplastic response under uniaxial loading-unloading conditions.

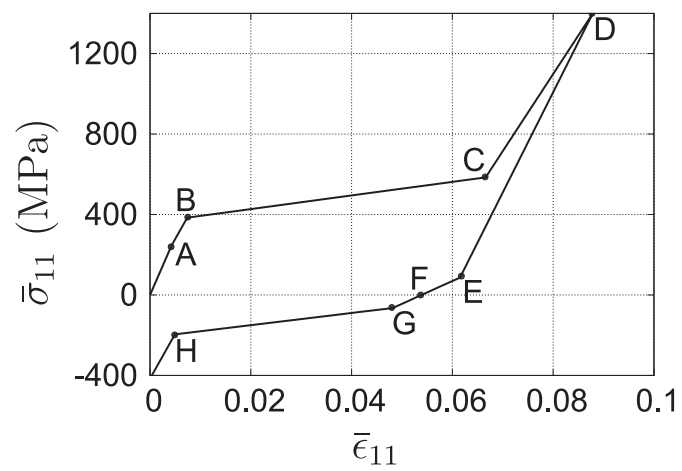

\begin{tabular}{|c|c|c|}
\hline \multicolumn{3}{|c|}{$\begin{array}{l}\text { Point F: zero macro-stress level during } \\
\text { unloading (reverse transformation) }\end{array}$} \\
\hline micro-stress (MPa) & SMA & Metallic material \\
\hline$\sigma_{11}$ & 0 & 0 \\
\hline$\sigma_{22}$ & -209.91 & 839.64 \\
\hline$\sigma_{33}$ & -209.91 & 839.64 \\
\hline
\end{tabular}

Figure 10. Mutilayered composite with $80 \%$ SMA volume fraction: Composite response under pure tension macro-loading and RVE response at zero macro-stress during unloading.

\subsection{Laminate with SMA and elastoplastic material}

In the second series of examples the metallic material presents elastoplastic Von-Mises response with a linear isotropic hardening. Its elastic properties remain the same, while for the plastic behavior a yield stress $275 \mathrm{MPa}$ and a linear hardening $12 \mathrm{GPa}$ are assumed. The uniaxial macroscopic stress strain curve of the pure metallic material is shown in figure 9 .

The following analysis considers zero macroscopic shear loading. The elastoplastic constitutive law of the matallic material is described through a convex cutting plane return mapping algorithm (Simo and Hughes 1998). In figure 10 the overall response of the composite with $80 \%$ SMA and $20 \%$ metallic material is presented. At the initial step the composite is loaded in tension and both the SMA and the metallic material are behaving elastically. At point $\mathrm{A}$ of the curve the metallic material starts developing plastic strains and there is a slight change in the slope of the curve. At point B the SMA

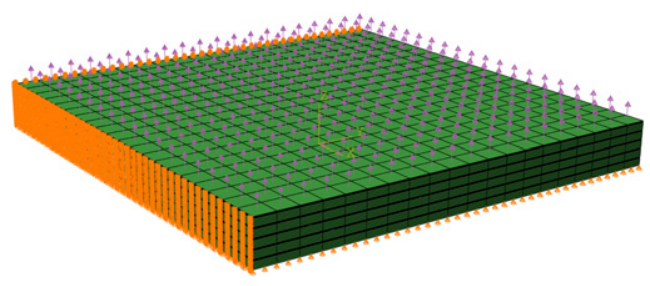

Figure 11. Laminate composite with $80 \%$ SMA volume fraction and $n=5$ (5 SMA layers and 5 metallic material layers). Finite element mesh in ABAQUS. The normal displacements at the bottom, left, and behind surfaces are constrained (roller support), while the upper surface is subjected to traction.

starts transforming and this process continues until point $\mathrm{C}$, where all austenite has been transformed into martensite. Then until point D the SMA behaves elastically and the metallic material behaves plastically. During unloading the metallic material behaves elastically, that is why two different slopes at point $\mathrm{D}$ are observed. At point $\mathrm{E}$ the metallic material continues to be in an elastic state but the SMA enters in the reverse transformation. At point $\mathrm{G}$ the metallic material enters again in the plastic region, while the SMA still transforms. The reverse transformation finishes at point $\mathrm{H}$ and the composite returns to zero macroscopic $\bar{\epsilon}_{11}$ strain when the macroscopic $\bar{\sigma}_{11}$ stress is approximately $400 \mathrm{MPa}$ in compression.

It must be noticed that at zero macro-stress level the SMA material is in a biaxial compression state (see table in figure 10). Thus, it is important to know if the SMA material response presents tension-compression asymmetry in order to obtain the correct overall response, even if the composite is loaded only under macroscopic tension conditions.

To verify the results of the homogenization method, a numerical analysis is performed for a composite laminate with $\mathrm{n}$ number of RVEs using the commercial finite element software ABAQUS. This computational procedure solves the whole composite (not only the RVE) using the macroscopic boundary conditions. Thus, it can be considered as the reference solution for the composite response, independently of any homogenization scheme.

According to the homogenization theory, as the number $\mathrm{n}$ increases, the average response of the composite should attain asymptotically the response predicted by the homogenization procedure. In the current study $n$ takes the values $3,4,5$, and 10. To facilitate the process, all boundary value problems for various $\mathrm{n}$ were generated through an appropriate python script. For $\mathrm{n}=5$, figure 11 shows the finite element mesh that consists of 20000 quadratic elements C3D20R in total. The boundary conditions of the composite are considered the macroscopic ones, i.e., uniform traction along the axis normal to the layers. The analysis in ABAQUS confirms the accuracy of the homogenization scheme. The finite element results appear to be insensitive to the number of RVEs and both the macroscopic response (figure 12) and the microscopic stress $\sigma_{22}$ in the SMA constituent (figure 13) are predicted correctly from the homogenization method. In the finite element analysis, $\sigma_{22}^{\mathrm{SMA}}$ refers to the average $\sigma_{22}$ stress component of an 


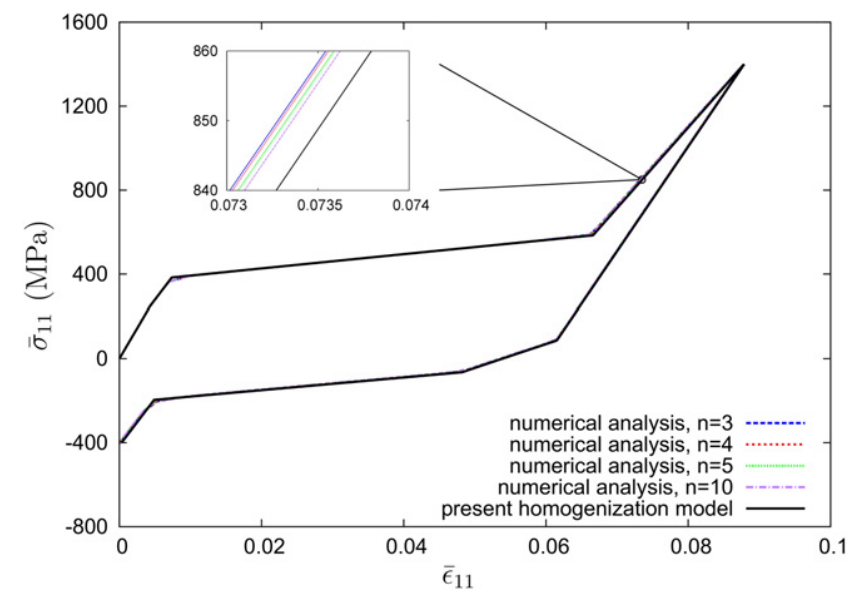

Figure 12. Laminate composite with $80 \%$ SMA volume fraction: Comparison between numerical analysis with ABAQUS and homogenization scheme. Macroscopic stress $\bar{\sigma}_{11}$ versus macroscopic strain $\bar{\epsilon}_{11}$.

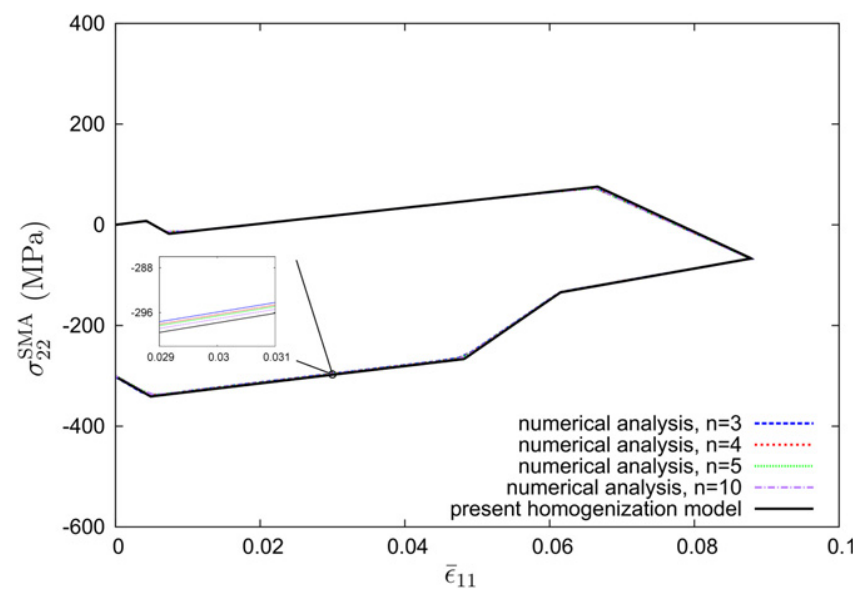

Figure 13. Mutilayered composite with $80 \%$ SMA volume fraction: Comparison between numerical analysis with ABAQUS and homogenization scheme. SMA stress $\sigma_{22}$ versus macroscopic strain $\bar{\epsilon}_{11}$.

arbitrary SMA layer ${ }^{2}$. Thus, the large computational cost of the finite element procedure (which is even higher for strongly nonlinear SMA laws) can be avoided by utilizing the described homogenization methodology, which allows us to obtain very accurately the actual composite response.

In the previous analysis the plastic yielding of the metallic material started earlier than the SMA transformation. In the next example the yield stress of the metallic material is tripled ( $825 \mathrm{MPa}$ ), while keeping the same composite synthesis and metallic material hardening slope. The resulting composite response is illustrated in figure 14. As in the previous example, at the initial step of the macro-tension loading both the SMA and the metallic material behave elastically. At point $\mathrm{A}^{\prime}$ of the macroscopic curve the SMA starts transforming and there is an important change in the slope of the curve. At point $\mathrm{B}$ ' the metallic material starts developing

\footnotetext{
${ }^{2}$ For consistency reasons, it always used the second SMA layer starting from the bottom.
}

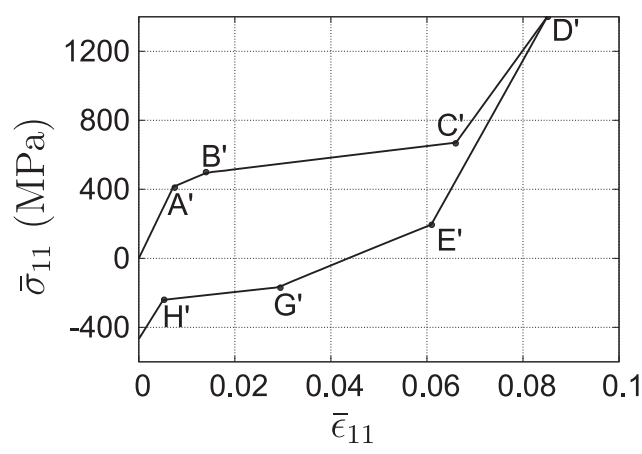

Figure 14. Mutilayered composite with $80 \%$ SMA volume fraction and increased metallic material yield stress: Composite response under pure tension macro-loading.

Table 2. Material properties of nickel-rich NiTi (Lagoudas et al 2012).

\begin{tabular}{lccc}
\hline$E^{A}(\mathrm{GPa})$ & $E^{M}(\mathrm{GPa})$ & $\nu^{A}$ & $\nu^{M}$ \\
90 & 63 & 0.3 & 0.3 \\
$H^{\text {min }}$ & $H^{\text {sat }}$ & $k(1 / \mathrm{MPa})$ & $\sigma^{\text {crit }}(\mathrm{MPa})$ \\
0 & 0.016 & 0.0075 & 12 \\
$M_{s}(\mathrm{~K})$ & $M_{f}(\mathrm{~K})$ & $A_{s}(\mathrm{~K})$ & $A_{f}(\mathrm{~K})$ \\
308 & 242 & 288 & 342 \\
$n_{1}$ & $n_{2}$ & $n_{3}$ & $n_{4}$ \\
0.6 & 0.2 & 0.2 & 0.3 \\
$C^{A}(\mathrm{MPa} / \mathrm{K})$ & $C^{M}(\mathrm{MPa} / \mathrm{K})$ & $\alpha(1 / \mathrm{K})$ & $\theta_{0}(\mathrm{~K})$ \\
16 & 10 & $1.0 \mathrm{E}-5$ & 400 \\
\hline
\end{tabular}

Table 3. Material properties of epoxy.

\begin{tabular}{lccc}
\hline$E(\mathrm{GPa})$ & $\nu$ & $\alpha(1 / \mathrm{K})$ & $\theta_{0}(\mathrm{~K})$ \\
3.85 & 0.4 & $4.4 \mathrm{E}-5$ & 400 \\
\hline
\end{tabular}

plastic strains. At point C' all austenite has been transformed into martensite. Then, until the point D' the SMA behaves elastically and the metallic material behaves plastically. At the first steps of unloading the metallic material behaves elastically, which again causes the appearance of two different slopes at point D'. At point E' the metallic material continues to be in the elastic state but the SMA starts the reverse transformation. At point $G$ ' the metallic material enters again in the plastic region, while the SMA is still under transformation, until it finishes at point $\mathrm{H}^{\prime}$.

\section{Composite laminate with SMA constituents: isobaric case}

In the following example, composite laminates are studied under a quasi-static thermomechanical cycle. Since the velocities are considered very low, latent heat and heat transfer effects will be ignored. The examined composites consist of nickel-rich NiTi, whose properties are summarized in table 2, and epoxy with properties given in table 3 . The numerical example starts at temperature $400 \mathrm{~K}$ and the followed loading path has 4 steps: 


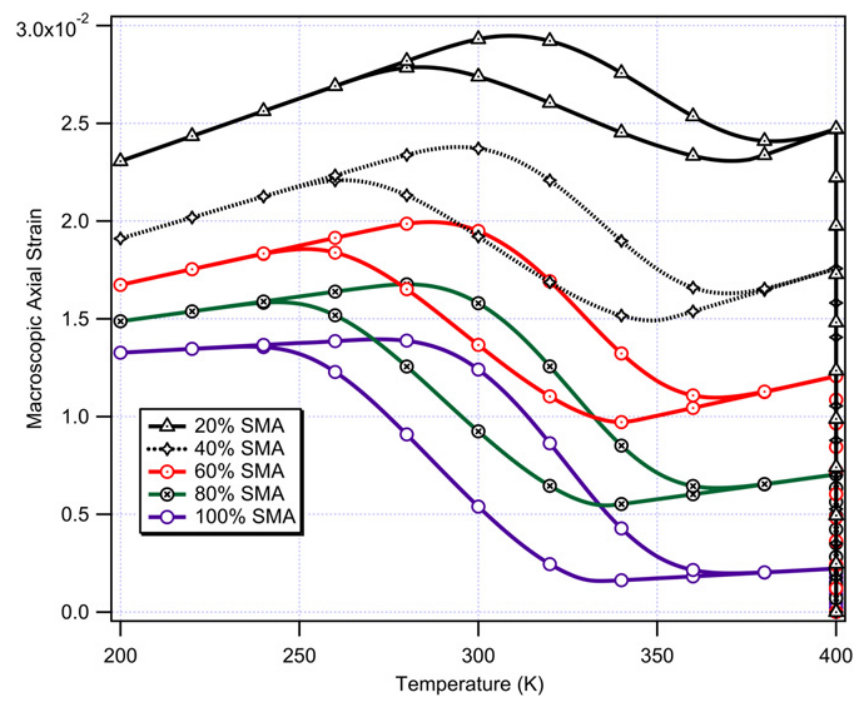

Figure 15. Macroscopic strain $\bar{\epsilon}_{11}$-temperature curves for pure SMA and composites with various SMA volume fraction during a complete thermomechanical loading cycle.

1. loading up to a stress level of $200 \mathrm{MPa}$ in the direction 1 ,

2. cooling until the temperature reaches $200 \mathrm{~K}$,

3. heating until the temperature reaches $400 \mathrm{~K}$, and

4. unloading to zero stress level.

The strain-temperature diagram of the analysis is illustrated in figure 15 for pure SMA and for composites with 20, 40, 60 and $80 \%$ epoxy. The results indicate that the increase of the epoxy volume fraction increases the strain levels and decreases the maximum transformation strain of the composite. Also the transformation temperatures increase significantly at low SMA volume fractions, which indicates that the stress level inside the alloy has increased substantially.

\section{Summary and conclusions}

The following points summarize this paper's goals:

- This work introduced a computational homogenization approach, based on the periodic homogenization theory, for composites with SMA constituents. The proposed methodology identifies the macro- and micro-responses of the composites under quasi-static thermomechanical conditions, independently of the micro-geometry and the macroscopic boundary conditions.

- The proposed homogenization framework was tested in the case of composite laminates, where semi-analytical solutions are available, and both the macro and microresponses showed excellent agreement with those obtained by finite element analysis performed in structures with multiple RVEs. This agreement is practically insensitive to the number of RVEs of the structure, thus the current model can be used for laminate materials with small number of layers. The proposed method allows us to avoid the computational cost of the FE analysis, which is enlarged by the complexity of the SMA constitutive law, without reducing the accuracy in the prediction of the composite response.

The numerical examples on the current study illustrated that even for simple uniaxial macroscopic boundary conditions the SMA components can be in triaxial stress state. Thus, to capture accurately the behavior of the composite, it is very important to account for possible anisotropic behavior of the SMA constituents, as well as other type of mechanisms like the martensitic variant reorientation. Computations on composite laminates with SMA and metallic material (elastic) components revealed that the transformation criteria of the composite can be significantly different from that of a typical SMA. An interesting observation is that at the finish of reverse transformation a non-convex macroscopic response appeared at high metallic material content. The results for multilayered materials with SMA and metallic material (elastoplastic) components showed complex macroscopic response, with significant interactions between the transformation and the plasticity. The sequence of the appearance of the nonlinear micro-mechanisms depends on the yield stress of the metallic material and the activation stresses of the SMA (start and finish of forward and reverse transformation). Finally, thermomechanical loading paths on composite laminates indicate that the presence of elastic components can change drastically the transformation temperatures at specific stress levels, compared to the pure SMA behavior.

Future extensions of this work could include analyses of SMA composites with more complicated microstructures (particle, fiber, woven composites), as well as the incorporation of other type of nonlinear mechanisms (for instance, damage). Additionally, the interplay between the mechanical and thermal effects will be examined through a fully coupled thermomechanical analysis.

\section{Acknowledgments}

The authors would like to thank Ali Javili (Chair of Applied Mechanics, Friedrich-Alexander University of ErlangenNuremberg) for helpful discussion.

\section{Appendix A. Numerical algorithms for periodic homogenization}

The numerical algorithms presented in this appendix have similar structure with those presented in Tsalis et al 2013 for the case of elastoplastic media with generalized periodicity. The symbol $\zeta$ denotes the internal variables of the material constituents that appear in an RVE (in an elastoplastic material these can be the plastic strain tensor and the equivalent plastic strain, in a shape memory alloy these can be the transformation strain tensor and the martensitic volume fraction). In table A2 the constitutive law algorithm can be a classical return mapping algorithm that computes the stress, 
Table A1. Macroscale analysis algorithm.

1. At time step $n$ everything is known. At time step $n+1$ and iteration step $m=0$ set $\Delta \overline{\boldsymbol{u}}^{(n+1)(0)}=\mathbf{0}, \overline{\boldsymbol{\epsilon}}^{(n+1)(0)}=\overline{\boldsymbol{\epsilon}}^{(n)}$, $\overline{\boldsymbol{\sigma}}^{(n+1)(0)}=\overline{\boldsymbol{\sigma}}^{(n)}, \Delta \bar{\theta}^{(n+1)}=\bar{\theta}^{(n+1)}-\bar{\theta}^{(n)}$.

2. In an RVE at each macroscopic point call the effective tangent moduli algorithm and obtain $\overline{\boldsymbol{D}}^{\epsilon(n+1)(m)}, \overline{\boldsymbol{D}}^{\theta(n+1)(m)}$.

3. Compute the virtual increment of $\overline{\boldsymbol{u}}$ from the macro-equilibrium equation

$$
\begin{gathered}
\int_{\bar{B}}^{\mathrm{grad}} \overline{\boldsymbol{\eta}}:\left[\overline{\boldsymbol{D}}^{\epsilon(n+1)(m)}: \overline{\operatorname{grad}} \delta \overline{\boldsymbol{u}}^{(n+1)(m)}+\overline{\boldsymbol{\sigma}}^{(n+1)(m)}\right] \mathrm{d} V \\
-\int_{\partial \overline{\bar{B}}^{\mathrm{NB}}} \overline{\boldsymbol{\eta}} \cdot \overline{\boldsymbol{t}}^{(n+1)} \mathrm{d} S=0 .
\end{gathered}
$$

4. Update the macro-quantities

$\Delta \overline{\boldsymbol{u}}^{(n+1)(m+1)}=\Delta \overline{\boldsymbol{u}}^{(n+1)(m)}+\delta \overline{\boldsymbol{u}}^{(n+1)(m)}$,

$\delta \overline{\boldsymbol{\epsilon}}^{(n+1)(m)}=\operatorname{grad}_{\mathrm{sym}} \delta \overline{\boldsymbol{u}}^{(n+1)(m)}$,

$\overline{\boldsymbol{\epsilon}}^{(n+1)(m+1)}=\overline{\boldsymbol{\epsilon}}^{(n+1)(m)}+\delta \overline{\boldsymbol{\epsilon}}^{(n+1)(m)}$

5. At each macroscopic point, call the RVE problem algorithm and compute $\overline{\boldsymbol{\sigma}}^{(n+1)(m+1)}$.

6. If $\underbrace{\max }_{\bar{V}}\left\|\delta \overline{\boldsymbol{u}}^{(n+1)(m)}\right\| /\left\|\Delta \overline{\boldsymbol{u}}^{(n+1)(m+1)}\right\| \leqslant \overline{\text { tol }}$

then compute the macro-displacement $\overline{\boldsymbol{u}}^{(n+1)}=\overline{\boldsymbol{u}}^{(n)}+\Delta \overline{\boldsymbol{u}}^{(n+1)}$, update the values of $\boldsymbol{\epsilon}^{(n+1)}, \boldsymbol{\zeta}^{(n+1)}$ of the RVE problem, set $n=n+1$ and return to step 1 ,

else set $m=m+1$ and return to step 2 .

the tangent thermomechanical moduli, and the change of the internal variables for given strain and initial values of $\zeta$ (see Simo and Hughes 1998 for elastoplastic or viscoplastic materials and Lagoudas 2008 for SMAs) .

\section{Appendix B. Solution of the RVE and the effective mechanical tangent modulus problems for a composite laminate}

Similar solution strategies for composite laminates with linear material constituents, using the periodic homogenization method, have been presented in the literature by several authors (see for instance Kalamkarov and Kolpakov 1997 in the case of laminated plates). For the completeness of the manuscript, this appendix presents the semi-analytical solution for the RVE and the tangent moduli problems. The effective thermal tangent modulus is omitted, because it was not utilized in the performed analyses, but its computation follows similar steps with the mechanical one.

A composite laminate, whose RVE consists of $N$ constituents, is demonstrated in figure B1. Each constituent has volume fraction $c^{(k)}$ and mechanical tangent modulus $\boldsymbol{D}^{\epsilon(k)}$ $(k=1,2, \ldots, N)$. Obviously $\sum_{r=1}^{N} c^{(r)}=1$. For simplicity, in all the subsequent expressions the iteration step is omitted and only the material that the quantity refers to is defined.

\section{RVE problem}

In the RVE of the multilayered material and under periodicity conditions the periodic part of the displacement, $\delta z$, is uniform along the $x_{2}$ and $x_{3}$ axes and presents non-uniformity only on the $x_{1}$ axis. Thus, equation (22) can be substituted with its local form for each material $k(k=1,2, \ldots, N)$, which is the one-dimensional differential equation system

$$
\frac{\mathrm{d}}{\mathrm{d} x_{1}}\left(\boldsymbol{D}_{n n}^{\epsilon(k)} \cdot \frac{\mathrm{d} \delta \boldsymbol{z}^{(k)}}{\mathrm{d} x_{1}}+\boldsymbol{\sigma}_{n}^{(k)}\right)=\mathbf{0},
$$

with

$$
\boldsymbol{D}_{n n}^{\epsilon(k)}=\left[\begin{array}{ccc}
D_{1111}^{\epsilon(k)} & D_{1121}^{\epsilon(k)} & D_{1131}^{\epsilon(k)} \\
D_{2111}^{\epsilon(k)} & D_{2121}^{\epsilon(k)} & D_{2131}^{\epsilon(k)} \\
D_{3111}^{\epsilon(k)} & D_{3121}^{\epsilon(k)} & D_{3131}^{\epsilon(k)}
\end{array}\right] \text { and } \boldsymbol{\sigma}_{n}^{(k)}=\left[\begin{array}{c}
\sigma_{11}^{(k)} \\
\sigma_{21}^{(k)} \\
\sigma_{31}^{(k)}
\end{array}\right]
$$

Due to the continuity of tractions, the system (B.1) after integration is written

$$
\begin{aligned}
& \boldsymbol{D}_{n n}^{\epsilon(k)} \cdot \frac{\mathrm{d} \delta z^{(k)}}{\mathrm{d} x_{1}}+\boldsymbol{\sigma}_{n}^{(k)}=\boldsymbol{m}, \quad \text { or } \\
& \frac{\mathrm{d} \delta z^{(k)}}{\mathrm{d} x_{1}}=\left[\boldsymbol{D}_{n n}^{\epsilon(k)}\right]^{-1} \cdot\left[\boldsymbol{m}-\boldsymbol{\sigma}_{n}^{(k)}\right],
\end{aligned}
$$

where $\boldsymbol{m}$ is a constant vector independent of the material. Integrating once more the solution of the system is obtained in the form

$$
\delta \boldsymbol{z}^{(k)}=\left[\boldsymbol{D}_{n n}^{\epsilon(k)}\right]^{-1} \cdot\left[\boldsymbol{m}-\boldsymbol{\sigma}_{n}^{(k)}\right] x_{1}+\boldsymbol{e}^{(k)},
$$

where $\boldsymbol{e}^{(k)}$ are constant vectors that depend on the material. The final expression indicates that $\delta z^{(k)}$ are linear functions of $x_{1}$. Considering periodicity of the vector $\delta z^{(k)}$ it can be assumed, without loss of generality, that $\delta \boldsymbol{z}^{(1)}(0)=\delta \boldsymbol{z}^{(N)}(1)=\mathbf{0}$, which leads to

$$
\boldsymbol{e}^{(1)}=\mathbf{0}, \quad \boldsymbol{e}^{(N)}=-\left[\boldsymbol{D}_{n n}^{\epsilon(N)}\right]^{-1} \cdot\left[\boldsymbol{m}-\boldsymbol{\sigma}_{n}^{(N)}\right] .
$$

The continuity of $\delta z^{(k)}$ at all the interfaces is expressed by the relation

$$
\delta z^{(k)}\left(c^{(k)}\right)=\delta z^{(k+1)}\left(c^{(k)}\right), \quad k=1,2, \ldots, N-1 .
$$

Using equations (B.4), (B.6) is expressed for each interface as

$$
\begin{aligned}
& {\left[\boldsymbol{D}_{n n}^{\epsilon(1)}\right]^{-1} \cdot\left[\boldsymbol{m}-\boldsymbol{\sigma}_{n}^{(1)}\right] c^{(1)}=\left[\boldsymbol{D}_{n n}^{\epsilon(2)}\right]^{-1}} \\
& \cdot\left[\boldsymbol{m}-\boldsymbol{\sigma}_{n}^{(2)}\right] c^{(1)}+\boldsymbol{e}^{(2)}, \\
& {\left[\boldsymbol{D}_{n n}^{\epsilon(2)}\right]^{-1} \cdot\left[\boldsymbol{m}-\boldsymbol{\sigma}_{n}^{(2)}\right]\left[c^{(1)}+c^{(2)}\right]+\boldsymbol{e}^{(2)}} \\
& =\left[\boldsymbol{D}_{n n}^{\epsilon(3)}\right]^{-1} \cdot\left[\boldsymbol{m}-\boldsymbol{\sigma}_{n}^{(3)}\right]\left[c^{(1)}+c^{(2)}\right]+\boldsymbol{e}^{(3)}, \\
& {\left[\boldsymbol{D}_{n n}^{\epsilon(3)}\right]^{-1} \cdot\left[\boldsymbol{m}-\boldsymbol{\sigma}_{n}^{(3)}\right]\left[c^{(1)}+c^{(2)}+c^{(3)}\right]+\boldsymbol{e}^{(3)}} \\
& =\left[\boldsymbol{D}_{n n}^{\epsilon(4)}\right]^{-1} \cdot\left[\boldsymbol{m}-\boldsymbol{\sigma}_{n}^{(4)}\right]\left[c^{(1)}+c^{(2)}+c^{(3)}\right]+\boldsymbol{e}^{(4)},
\end{aligned}
$$


Table A2. RVE problem algorithm.

1.

2.

3.

4.

5.

6.

7.
At macroscale step $m+1$ and time step $n+1, \overline{\boldsymbol{\epsilon}}^{(n+1)(m+1)}$

and $\bar{\theta}^{(n+1)}$ are known.

At microscale iteration step $m^{*}=0$ set $\Delta \boldsymbol{z}^{(n+1)(m+1)(0)}=\mathbf{0}$, $\boldsymbol{\epsilon}^{(n+1)(m+1)(0)}=\boldsymbol{\epsilon}^{(n)}+\overline{\boldsymbol{\epsilon}}^{(n+1)(m+1)}-\overline{\boldsymbol{\epsilon}}^{(n)}, \boldsymbol{\zeta}^{(n+1)(m+1)(0)}=\boldsymbol{\zeta}^{(n)}$

Evaluate the micro-stresses $\boldsymbol{\sigma}^{(n+1)(m+1)(0)}$ and the micro-tangent thermomechanical moduli $\boldsymbol{D}^{\epsilon(n+1)(m+1)(0)}, \boldsymbol{D}^{\theta(n+1)(m+1)(0)}$

using the constitutive law algorithm.

Compute the virtual increment of $z$ from the micro-equilibrium equation $\int_{\mathcal{B}} \operatorname{grad} \boldsymbol{\eta}: \boldsymbol{D}^{\epsilon(n+1)(m+1)\left(m^{*}\right)}: \operatorname{grad} \delta \boldsymbol{z}^{(n+1)(m+1)\left(m^{*}\right)} \mathrm{d} V$

$$
+\int_{\mathcal{B}} \operatorname{grad} \boldsymbol{\eta}: \boldsymbol{\sigma}^{(n+1)(m+1)\left(m^{*}\right)} \mathrm{d} V=0 .
$$

Update the micro-quantities

$$
\begin{aligned}
& \Delta \boldsymbol{z}^{(n+1)(m+1)\left(m^{*}+1\right)}=\Delta \boldsymbol{z}^{(n+1)(m+1)\left(m^{*}\right)}+\delta \boldsymbol{z}^{(n+1)(m+1)\left(m^{*}\right)}, \\
& \delta \boldsymbol{\epsilon}^{(n+1)(m+1)\left(m^{*}\right)}=\operatorname{grad}_{\mathrm{sym}} \delta \boldsymbol{z}^{(n+1)(m+1)\left(m^{*}\right)}, \\
& \boldsymbol{\epsilon}^{(n+1)(m+1)\left(m^{*}+1\right)}=\boldsymbol{\epsilon}^{(n+1)(m+1)\left(m^{*}\right)}+\delta \boldsymbol{\epsilon}^{(n+1)(m+1)\left(m^{*}\right)} .
\end{aligned}
$$

At each microscopic point in the RVE, obtain the micro-stresses $\boldsymbol{\sigma}^{(n+1)(m+1)\left(m^{*}+1\right)}$ and the micro-tangent moduli $\boldsymbol{D}^{\epsilon(n+1)(m+1)(0)}, \boldsymbol{D}^{\theta(n+1)(m+1)(0)}$

using the constitutive law algorithm.

If $\underbrace{\max }_{V}\left\|\delta z^{(n+1)(m+1)\left(m^{*}\right)}\right\| /\left\|\Delta z^{(n+1)(m+1)\left(m^{*}+1\right)}\right\| \leqslant$ tol

then continue with step 6 ,

else set $m^{*}=m^{*}+1$ and return to step 2 .

Compute the periodic part of the micro-displacement,

$\boldsymbol{z}^{(n+1)(m+1)}=\boldsymbol{z}^{(n)}+\Delta \boldsymbol{z}^{(n+1)(m+1)}$.

Compute the macro-stress, $\overline{\boldsymbol{\sigma}}^{(n+1)(m+1)}=\frac{1}{V} \int_{\mathcal{B}} \boldsymbol{\sigma}^{(n+1)(m+1)} \mathrm{d} V$.
Table A3. Effective tangent moduli algorithm.

1. Using the tangent moduli at each point of the RVE, known from the last solution of the RVE problem algorithm,

compute the correctors $\chi^{\epsilon}$ and $\chi^{\theta}$ from the equations

$\int_{B} \operatorname{grad} \boldsymbol{\eta}:\left[\boldsymbol{D}^{\epsilon}: \operatorname{grad} \chi^{\epsilon}+\boldsymbol{D}^{\epsilon}\right] \mathrm{d} V=0$,

$\int_{B} \operatorname{grad} \boldsymbol{\eta}:\left[\boldsymbol{D}^{\epsilon}: \operatorname{grad} \chi^{\theta}+\boldsymbol{D}^{\theta}\right] \mathrm{d} V=0$.

2. Evaluate the effective tangent moduli

$\overline{\boldsymbol{D}}^{\epsilon}=\frac{1}{V} \int_{B}\left[\boldsymbol{D}^{\epsilon} \tilde{:} \operatorname{grad} \chi^{\epsilon}+\boldsymbol{D}^{\epsilon}\right] \mathrm{d} V$,

$\overline{\boldsymbol{D}}^{\theta}=\frac{1}{V} \int_{B}\left[\boldsymbol{D}^{\epsilon}: \operatorname{grad} \chi^{\theta}+\boldsymbol{D}^{\theta}\right] \mathrm{d} V$.

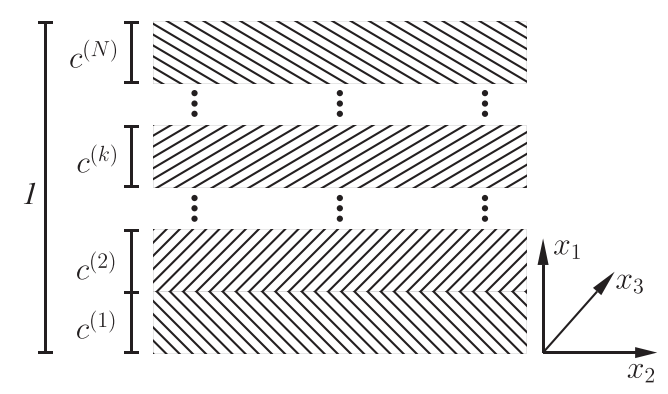

Figure B1. Schematic representation of the RVE of a composite laminate with $N$ layers.

$$
\begin{aligned}
& {\left[\boldsymbol{D}_{n n}^{\epsilon(k)}\right]^{-1} \cdot\left[\boldsymbol{m}-\boldsymbol{\sigma}_{n}^{(k)}\right] \sum_{r=1}^{k} c^{(r)}+\boldsymbol{e}^{(k)}} \\
& =\left[\boldsymbol{D}_{n n}^{\epsilon(k+1)}\right]^{-1} \cdot\left[\boldsymbol{m}-\boldsymbol{\sigma}_{n}^{(k+1)}\right] \sum_{r=1}^{k} c^{(r)}+\boldsymbol{e}^{(k+1)}, \\
& {\left[\boldsymbol{D}_{n n}^{\epsilon(k+1)}\right]^{-1} \cdot\left[\boldsymbol{m}-\boldsymbol{\sigma}_{n}^{(k+1)}\right] \sum_{r=1}^{k+1} c^{(r)}+\boldsymbol{e}^{(k+1)}} \\
& =\left[\boldsymbol{D}_{n n}^{\epsilon(k+2)}\right]^{-1} \cdot\left[\boldsymbol{m}-\boldsymbol{\sigma}_{n}^{(k+2)}\right] \sum_{r=1}^{k+1} c^{(r)}+\boldsymbol{e}^{(k+2)}, \\
& {\left[\boldsymbol{D}_{n n}^{\epsilon(N-1)}\right]^{-1} \cdot\left[\boldsymbol{m}-\boldsymbol{\sigma}_{n}^{(N-1)}\right] \sum_{r=1}^{N-1} c^{(r)}+\boldsymbol{e}^{(N-1)}} \\
& =\left[\boldsymbol{D}_{n n}^{\epsilon(N)}\right]^{-1} \cdot\left[\boldsymbol{m}-\boldsymbol{\sigma}_{n}^{(N)}\right] \sum_{r=1}^{N-1} c^{(r)}-\left[\boldsymbol{D}_{n n}^{\epsilon(N)}\right]^{-1} \\
& \cdot\left[\boldsymbol{m}-\boldsymbol{\sigma}_{n}^{(N)}\right] \cdot
\end{aligned}
$$

Adding all the previous expressions leads to

$$
\sum_{r=1}^{N}\left[\boldsymbol{D}_{n n}^{\epsilon(r)}\right]^{-1} \cdot\left[\boldsymbol{m}-\boldsymbol{\sigma}_{n}^{(r)}\right] c^{(r)}=\mathbf{0},
$$




$$
\boldsymbol{m}=\left[\sum_{r=1}^{N} c^{(r)}\left[\boldsymbol{D}_{n n}^{\epsilon(r)}\right]^{-1}\right]^{-1} \cdot\left[\sum_{r=1}^{N} c^{(r)}\left[\boldsymbol{D}_{n n}^{\epsilon(r)}\right]^{-1} \cdot \boldsymbol{\sigma}_{n}^{(r)}\right]
$$

Equation (B.3) indicates that the knowledge of the vector $\boldsymbol{m}$ is sufficient to compute the increments of the micro-strains at each material, since

$$
\begin{gathered}
\delta \epsilon_{11}^{(k)}=\frac{\mathrm{d} \delta z_{1}^{(k)}}{\mathrm{d} x_{1}}, \quad \delta \epsilon_{21}^{(k)}=\frac{1}{2} \frac{\mathrm{d} \delta z_{2}^{(k)}}{\mathrm{d} x_{1}}, \quad \delta \epsilon_{31}^{(k)}=\frac{1}{2} \frac{\mathrm{d} \delta z_{3}^{(k)}}{\mathrm{d} x_{1}}, \\
\delta \epsilon_{k l}^{(k)}=0 \quad \text { for the rest of the cases. }
\end{gathered}
$$

With these increments we obtain the actual values of the micro-strain at each material at the specific iteration step.

\section{Effective mechanical tangent modulus}

For the simplicity of the subsequent expressions, it is easier to represent the symmetric, fourth-order, mechanical tangent modulus tensor of a material with the help of four secondorder tensors (Qu and Cherkaoui 2006),

$$
\begin{aligned}
\boldsymbol{D}_{n n}^{\epsilon} & =\left[\begin{array}{lll}
D_{1111}^{\epsilon} & D_{1121}^{\epsilon} & D_{1131}^{\epsilon} \\
D_{2111}^{\epsilon} & D_{2121}^{\epsilon} & D_{2131}^{\epsilon} \\
D_{3111}^{\epsilon} & D_{3121}^{\epsilon} & D_{3131}^{\epsilon}
\end{array}\right], \\
\boldsymbol{D}_{t n}^{\epsilon} & =\left[\begin{array}{lll}
D_{2211}^{\epsilon} & D_{2221}^{\epsilon} & D_{2231}^{\epsilon} \\
D_{3311}^{\epsilon} & D_{3321}^{\epsilon} & D_{3331}^{\epsilon} \\
D_{2311}^{\epsilon} & D_{2321}^{\epsilon} & D_{2331}^{\epsilon}
\end{array}\right], \\
\boldsymbol{D}_{n t}^{\epsilon} & =\left[\begin{array}{lll}
D_{1122}^{\epsilon} & D_{1133}^{\epsilon} & D_{1123}^{\epsilon} \\
D_{2122}^{\epsilon} & D_{2133}^{\epsilon} & D_{2123}^{\epsilon} \\
D_{3122}^{\epsilon} & D_{3133}^{\epsilon} & D_{3123}^{\epsilon}
\end{array}\right], \\
\boldsymbol{D}_{t t}^{\epsilon} & =\left[\begin{array}{lll}
D_{2222}^{\epsilon} & D_{2233}^{\epsilon} & D_{2223}^{\epsilon} \\
D_{3322}^{\epsilon} & D_{3333}^{\epsilon} & D_{3323}^{\epsilon} \\
D_{2322}^{\epsilon} & D_{2333}^{\epsilon} & D_{2323}^{\epsilon}
\end{array}\right],
\end{aligned}
$$

Following similar steps with the RVE problem, equation (25) is substituted with its local form for each material $k$, and takes the following two forms,

$$
\begin{aligned}
& \frac{\mathrm{d}}{\mathrm{d} x_{1}}\left(\boldsymbol{D}_{n n}^{\epsilon(k)} \cdot \frac{\mathrm{d} \chi_{n}^{\epsilon(k)}}{\mathrm{d} x_{1}}+\boldsymbol{D}_{n n}^{\epsilon(k)}\right)=\mathbf{0}, \\
& \frac{\mathrm{d}}{\mathrm{d} x_{1}}\left(\boldsymbol{D}_{n n}^{\epsilon(k)} \cdot \frac{\mathrm{d} \chi_{t}^{\epsilon(k)}}{\mathrm{d} x_{1}}+\boldsymbol{D}_{n t}^{\epsilon(k)}\right)=\mathbf{0},
\end{aligned}
$$

with

$$
\begin{aligned}
& \chi_{n}^{\epsilon(k)}=\left[\begin{array}{lll}
\chi_{111}^{\epsilon(k)} & \chi_{121}^{\epsilon(k)} & \chi_{131}^{\epsilon(k)} \\
\chi_{111}^{\epsilon(k)} & \chi_{221}^{\epsilon \epsilon()} & \chi_{231}^{\epsilon(k)} \\
\chi_{311}^{\epsilon(k)} & \chi_{321}^{\epsilon(k)} & \chi_{331}^{\epsilon(k)}
\end{array}\right], \\
& \chi_{t}^{\epsilon(k)}=\left[\begin{array}{lll}
\chi_{122}^{\epsilon(k)} & \chi_{133}^{\epsilon(k)} & \chi_{123}^{\epsilon(k)} \\
\chi_{222}^{\epsilon(k)} & \chi_{233}^{\epsilon \epsilon(} & \chi_{223}^{\epsilon(k)} \\
\chi_{322}^{\epsilon(k)} & \chi_{333}^{\epsilon(k)} & \chi_{323}^{\epsilon(k)}
\end{array}\right] .
\end{aligned}
$$

It is recalled that the micro-tangent moduli $\boldsymbol{D}^{(k)}$ used here are the same with the micro-tangent moduli at the last iteration step of the RVE problem. The systems (B.10) have exactly the same structure as equation (B.1), thus its solution has similar form with equation (B.4), i.e.,

$$
\begin{aligned}
\frac{\mathrm{d} x_{n}^{\epsilon(k)}}{\mathrm{d} x_{1}}= & {\left[\boldsymbol{D}_{n n}^{\epsilon(k)}\right]^{-1} \cdot\left[\boldsymbol{m}_{n}-\boldsymbol{D}_{n n}^{\epsilon(k)}\right], } \\
\frac{\mathrm{d} x_{t}^{\epsilon(k)}}{\mathrm{d} x_{1}}= & {\left[\boldsymbol{D}_{n n}^{\epsilon(k)}\right]^{-1} \cdot\left[\boldsymbol{m}_{t}-\boldsymbol{D}_{n t}^{\epsilon(k)}\right], } \\
\boldsymbol{m}_{n}= & {\left[\sum_{r=1}^{N} c^{(r)}\left[\boldsymbol{D}_{n n}^{\epsilon(r)}\right]^{-1}\right]^{-1} } \\
& \cdot\left[\sum_{r=1}^{N} c^{(r)}\left[\boldsymbol{D}_{n n}^{\epsilon(r)}\right]^{-1} \cdot \boldsymbol{D}_{n n}^{\epsilon(r)}\right] \\
= & {\left[\sum_{r=1}^{N} c^{(r)}\left[\boldsymbol{D}_{n n}^{\epsilon(r)}\right]^{-1}\right]^{-1}, } \\
\boldsymbol{m}_{t}= & {\left[\sum_{r=1}^{N} c^{(r)}\left[\boldsymbol{D}_{n n}^{\epsilon(r)}\right]^{-1}\right]^{-1} } \\
& \cdot\left[\sum_{r=1}^{N} c^{(r)}\left[\boldsymbol{D}_{n n}^{\epsilon(r)}\right]^{-1} \cdot \boldsymbol{D}_{n t}^{\epsilon(r)}\right] .
\end{aligned}
$$

Thus, according to equation (28), the effective mechanical tangent modulus of the composite laminate is expressed as

$$
\begin{aligned}
\overline{\boldsymbol{D}}_{n n}^{\epsilon}= & \sum_{r=1}^{N} c^{(r)}\left[\boldsymbol{D}_{n n}^{\epsilon(r)} \cdot \frac{\mathrm{d} \chi_{n}^{\epsilon(r)}}{\mathrm{d} x_{1}}+\boldsymbol{D}_{n n}^{\epsilon(r)}\right]= \\
\boldsymbol{m}_{n}= & {\left[\sum_{r=1}^{N} c^{(r)}\left[\boldsymbol{D}_{n n}^{\epsilon(r)}\right]^{-1}\right]^{-1}, } \\
\overline{\boldsymbol{D}}_{n t}^{\epsilon}= & \sum_{r=1}^{N} c^{(r)}\left[\boldsymbol{D}_{n n}^{\epsilon(r)} \cdot \frac{\mathrm{d} \chi_{t}^{\epsilon(r)}}{\mathrm{d} x_{1}}+\boldsymbol{D}_{n t}^{\epsilon(r)}\right]=\boldsymbol{m}_{t} \\
= & \overline{\boldsymbol{D}}_{n n}^{\epsilon} \cdot\left[\sum_{r=1}^{N} c^{(r)}\left[\boldsymbol{D}_{n n}^{\epsilon(r)}\right]^{-1} \cdot \boldsymbol{D}_{n t}^{\epsilon(r)}\right], \\
\overline{\boldsymbol{D}}_{t n}^{\epsilon}= & \sum_{r=1}^{N} c^{(r)}\left[\boldsymbol{D}_{t n}^{\epsilon(r)} \cdot \frac{\mathrm{d} \chi_{n}^{\epsilon(r)}}{\mathrm{d} x_{1}}+\boldsymbol{D}_{t n}^{\epsilon(r)}\right] \\
= & \sum_{r=1}^{N} c^{(r)}\left[\boldsymbol{D}_{t n}^{\epsilon(r)} \cdot\left[\boldsymbol{D}_{n n}^{\epsilon(r)}\right]^{-1} \cdot \boldsymbol{m}_{n}\right] \\
= & {\left[\sum_{r=1}^{N} c^{(r)} \boldsymbol{D}_{t n}^{\epsilon(r)} \cdot\left[\boldsymbol{D}_{n n}^{\epsilon(r)}\right]^{-1}\right] \cdot \overline{\boldsymbol{D}}_{n n}^{\epsilon}=\left[\overline{\boldsymbol{D}}_{n t}^{\epsilon}\right]^{\mathrm{T}}, } \\
\overline{\boldsymbol{D}}_{t t}^{\epsilon}= & \sum_{r=1}^{N} c^{(r)}\left[\boldsymbol{D}_{t n}^{\epsilon(r)} \cdot \frac{\mathrm{d} \chi_{t}^{\epsilon(r)}}{\mathrm{d} x_{1}}+\boldsymbol{D}_{t t}^{\epsilon(r)}\right] \\
= & \sum_{r=1}^{N} c^{(r)}\left[\boldsymbol{D}_{t n}^{\epsilon(r)} \cdot\left[\boldsymbol{D}_{n n}^{\epsilon(r)}\right]^{-1}\right. \\
& \left.\cdot\left[\overline{\boldsymbol{D}}_{n t}^{\epsilon}-\boldsymbol{D}_{n t}^{\epsilon(r)}\right]+\boldsymbol{D}_{t t}^{\epsilon(r)}\right] .
\end{aligned}
$$

In the case of elastic constituents, these formulas coincide with the ones provided by $\mathrm{Qu}$ and Cherkaoui (2006) for composite laminates using classical micromechanics techniques. 


\section{References}

Allaire G 1992 Homogenization and two-scale convergence SIAM J. Math. Anal. 23 1482-518

Anand L and Gurtin M E 2003 Thermal effects in the superelasticity of crystalline shape memory materials J. Mech. Phys. Solids 51 1015-58

Asada T and Ohno N 2007 Fully implicit formulation of elastoplastic homogenization problem for two-scale analysis Int. J. Solids Struct. 44 7261-75

Auricchio F, Taylor R L and Lubliner J 1997 Shape-memory alloys: macromodelling and numerical simulations of the superelastic behavior Comput. Methods Appl. Mech. Eng. 146 281-312

Baz A and Ro J 1992 Thermo-dynamic characteristics of nitinolreinforced composite beams Compos. Eng. 2 527-42

Bensoussan A, Lions J and Papanicolaou G 1978 Asymptotic methods for periodic structures (Amsterdam: North Holland)

Birman V 1997 Review of Mechanics of Shape Memory Alloy Structures Appl. Mech. Rev. 50 629-45

Boyd J G and Lagoudas D C 1994 Thermomechanical Response of Shape Memory Composites J. Intell. Mater. Syst. Struct. 5 333-46

Boyd J G and Lagoudas D C 1996 A thermodynamical constitutive model for shape memory materials. Part I. The monolithic shape memory alloy Int. J. Plast. 12 805-42

Brinson L C 1993 One-Dimensional Constitutive Behavior of Shape Memory Alloys: Thermomechanical Derivation with NonConstant Material Functions and Redefined Martensite Internal Variable J. Intell. Mater. Syst. Struct. 4 229-42

Cavalcante M A A, Khatam H and Pindera M J 2011 Homogenization of elastic-plastic periodic materials by FVDAM and FEM approaches-An assessment Composites Part B- Engineering 42 1713-30

Charalambakis N 2010 Homogenization Techniques and Micromechanics. A Survey and Perspectives Appl. Mech. Rev. 63030803

Chatzigeorgiou G, Javili A and Steinmann P 2013 Multiscale modeling for composites with energetic interfaces at the microor nanoscale Mathematics and Mechanics of Solids doi:10.1177/1081286513516122

Chemisky Y, Duval A, Patoor E and ben Zineb T 2011 Constitutive model for shape memory alloys including phase transformation, martensitic reorientation and twins accommodation Mech. Mater. 43 361-76

Cherkaoui M, Sun Q P and Song G Q 2000 Micromechanics modeling of composite with ductile matrix and shape memory alloy reinforcement Int. J. Solids Struct. 37 1577-94

Collard C, Ben Zineb T, Patoor E and Ben Salah M O 2008 Micromechanical analysis of precipitate effects on shape memory alloys behaviour Materials Science and Engineering: A 481-482 366-70

Desrumaux F, Meraghni F and Benzeggagh M L 2001 Generalised Mori-Tanaka Scheme to Model Anisotropic Damage Using Numerical Eshelby Tensor J. Compos. Mater. 35 603-24

Ene H I 1983 On linear thermoelasticity of composite materials Int. J. Eng. Sci. 21 443-8

Entchev P B and Lagoudas D C 2002 Modeling porous shape memory alloys using micromechanical averaging techniques Mech. Mater. 34 1-24

Entchev P B and Lagoudas D C 2004 Modeling of transformationinduced plasticity and its effect on the behavior of porous shape memory alloys: part II: porous SMA response Mech. Mater. 36 893-913

Geers M G D, Kouznetsova V G and Brekelmans W A M 2010 Multi-scale computational homogenization: Trends and challenges J. Comput. Appl. Math. 234 2175-82

Hartl D J, Chatzigeorgiou G and Lagoudas D C 2010 Threedimensional modeling and numerical analysis of rate- dependent irrecoverable deformation in shape memory alloys Int. J. Plast. 26 1485-507

Herzog H and Jacquet E 2007 From a shape memory alloys model implementation to a composite behavior Comput. Mater. Sci. 39 365-75

Hill R 1967 The essential structure of constitutive laws for metal composites and polycrystals J. Mech. Phys. Solids 15 79-95

Javili A, Chatzigeorgiou G and Steinmann P 2013 Computational homogenization in magneto-mechanics International Journal of Solids and Structures 50 4197-216

Jendli Z, Meraghni F, Fitoussi J and Baptist D 2009 Multi-scales modelling of dynamic behaviour for discontinuous fibre SMC composites Compos. Sci. Technol. 69 97-103

Kalamkarov A L and Kolpakov A G 1997 Analysis, design and optimization of composite structures (Chichester: Wiley)

Kanouté P, Boso D P, Chaboche J L and Schrefler B A 2009 Multiscale methods for composites: a review Archives of Computational Methods in Engineering 16 31-75

Kawai M, Ogawa H, Baburaj V and Koga T 1999 Micromechamical Analysis for Hysteretic Behavior of Unidirectional TiNi SMA Fiber Composites J. Intell. Mater. Syst. Struct. 10 14-28

Khatam H and Pindera M J 2009 Parametric finite-volume micromechanics of periodic materials with elastoplastic phases Int. J. Plast. 25 1386-411

Kruch S and Chaboche J L 2011 Multi-scale analysis in elastoviscoplasticity coupled with damage Int. J. Plast. 27 2026-39

Lagoudas D, Hartl D, Chemisky Y, Machado L and Popov P 2012 Constitutive model for the numerical analysis of phase transformation in polycrystalline shape memory alloys Int. $J$. Plast. 32-33 155-83

Lagoudas D C 2008 Shape Memory Alloys: Modeling and Engineering Applications (New York: Springer)

Lagoudas D C, Boyd J G and Bo Z 1994 Micromechanics of Active Composites With SMA Fibers Joumal of Engineering Materials and Technology 116 337-47

Lagoudas D C, Entchev P B, Popov P, Patoor E, Brinson L C and Gao X 2006 Shape Memory Alloys, Part II: modeling of polycrystals Mech. Mater. 38 430-62

Lagoudas D C, Moorthy D, Qidwai M A and Reddy J N 1997 Modeling of the thermomechanical response of active laminates with SMA strips using the layerwise finite element method J. Intell. Mater. Syst. Struct. 8 476-88

Lahellec N and Suquet P 2013 Effective response and field statistics in elasto-plastic and elasto-viscoplastic composites under radial and non-radial loadings Int. J. Plast. 42 1-30

Leclercq S and Lexcellent C 1996 A general macroscopic description of the thermomechanical behavior of shape memory alloys J. Mech. Phys. Solids 44 953-80

Lester B T, Chemisky Y and Lagoudas D C 2011 Transformation characteristics of shape memory alloy composites Smart Mater. Struct. 20094002

Liang C and Rogers C A 1990 One-Dimensional thermomechanical constitutive relations for shape memory materials $J$. Intell. Mater. Syst. Struct. 1 207-34

Maghous S and Creus G J 2003 Periodic homogenization in thermoviscoelasticity: case of multilayered media with ageing Int. J. Solids Struct. 40 851-70

Marfia S 2005 Micromacro analysis of shape memory alloy composites Int. J. Solids Struct. 4236773699

Meraghni F, Chemisky Y, Piotrowski B, Echchorfi R, Bourgeois N and Patoor E 2014 Parameter identification of a thermodynamic model for superelastic shape memory alloys using analytical calculation of the sensitivity matrix European Journal of Mechanics-A/Solids 45 226-37

Meraghni F, Desrumaux F and Benzeggagh M L 2002 Implementation of a constitutive micromechanical model for damage analysis in glass mat reinforced composite structures Compos. Sci. Technol. 62 2087-97 
Mercier S and Molinari A 2009 Homogenization of elasticviscoplastic heterogeneous materials: Self-consistent and MoriTanaka schemes Int. J. Plast. 25 1024-48

Murat F and Tartar L 1997 H-convergence, in Topics in the mathematical modelling of composite materials (Progress in Nonlinear Differential Equations and their Applications Vol 31) ed A Cherkaev and R Kohn 21-43 Birkhäuser

Nemat-Nasser S and Hori M 1999 Micromechanics: Overall Properties of Heterogeneous Materials 2nd Edition (Amsterdam: North-Holland)

Nemat-Nasser S, Sui Y, Guo W and Isaacs J 2005 Experimental characterization and micromechanical modeling of superelastic response of a porous NiTi shape-memory alloy Journal of the Mechanics and Physics of Solids 53 2320-46

Paiva A and Savi M A 2006 An overview of constitutive models for shape memory alloys Mathematical Problems in Engineering 2006 1-30

Paiva A, Savi M A, Braga A M B and Pacheco P M C L 2005 A constitutive model for shape memory alloys considering tensile-compressive assymetry and plasticity Int. J. Solids Struct. 42 3439-57

Panico M and Brinson L C 2008 Computational modeling of porous shape memory alloys Int. J. Solids Struct. 45 5613-26

Patoor E, Eberhardt A and Berveiller M 1996 Micromechanical Modelling of Superelasticity in Shape Memory Alloys Journal de Physique IV 6 277-92

Patoor E, Lagoudas D C, Entchev P B, Brinson L C and Gao X 2006 Shape memory alloys, part i: general properties and modeling of single crystals Mech. Mater. 38 391-429

Pindera M J, Khatam H, Drago A S and Bansal Y 2009

Micromechanics of spatially uniform heterogeneous media: A critical review and emerging approaches Composites Part B: Engineering 40 349-78

Piotrowski B, ben Zineb T, Patoor E and Eberhardt A 2012 Modeling of niobium precipitates effect on the $\mathrm{Ni}_{47} \mathrm{Ti}_{44} \mathrm{Nb}_{9}$ Shape Memory Alloy behavior Int. J. Plast. 36 130-47

Ponte-Castañeda P and Suquet P 1997 Nonlinear composites Advances in Applied Mechanics 34 171-302
Qidwai M A, Entchev P B, Lagoudas D C and DeGiorgi V G 2001 Modeling of the thermomechanical behavior of porous shape memory alloys Int. J. Solids Struct. 38 8653-71

Qu J and Cherkaoui M 2006 Fundamentals of Micromechanics of Solids (New Jersey: Wiley)

Ro J and Baz A 1995 NITINOL-reinforced plates: Part I. Thermal characteristics Compos. Eng. 5 61-75

Rogers C A, Liang C and Jia J 1991 Structural modification of simply-supported laminated plates using embedded shape memory alloy fibers Computers \& Structures 38 569-80

Sadjadpour A and Bhattacharya K 2007 A micromechanics-inspired constitutive model for shape-memory alloys Smart Mater. Struct. 16 1751-65

Sanchez-Palencia E 1980 Non-homogeneous media and vibration theory Lecture Notes Phys. 127

Simo J C and Hughes T J R 1998 Computational Inelasticity (New York: Springer)

Song G Q and Sun Q P 2000 Effect of microstructure on the mechanical behavior of NiTi shape memory alloy reinforced aluminum matrix composite Smart Mater. Struct. 9 693-700

Suquet P M 1987 Elements of homogenization for inelastic solid mechanics Lect. Notes Phys. 272 193-278

Tanaka K, Kobayashi S and Sato Y 1986 Thermomechanics of Transformation Pseudoelasticity and Shape Memory Effect in Alloys Int. J. Plast. 2 59-72

Tartar L 1978 Nonlinear constitutive relations and homogenization Contemporary Developments in Continuum Mechanics and Partial Differential Equations (Amsterdam: North-Holland)

Tekoğlu C and Pardoen T 2010 A micromechanics based damage model for composite materials Int. J. Plast. 26 549-69

Terada K and Kikuchi N 2001 A class of general algorithms for multi-scale analyses of heterogeneous media Comput. Methods Appl. Mech. Eng. 190 5427-64

Tsalis D, Baxevanis T, Chatzigeorgiou G and Charalambakis N 2013 Homogenization of elastoplastic composites with generalized periodicity in the microstructure Int. J. Plast. 51 161-87

Wang J and Shen Y P 2000 Micromechanics of composites reinforced in the aligned SMA short fibers in uniform thermal fields Smart Mater. Struct. 9 69-77 